\title{
Identification and characterization of Tc1/ mariner-like DNA transposons in genomes of the pathogenic fungi of the Paracoccidioides species complex
}

Marjorie M Marini ${ }^{1}$, Tamiris Zanforlin², Patrícia C Santos ${ }^{1}$, Roberto RM Barros ${ }^{2}$, Anne CP Guerra ${ }^{1}$, Rosana Puccia ${ }^{2}$, Maria SS Felipe ${ }^{3}$, Marcelo Brigido ${ }^{3}$, Célia MA Soares ${ }^{4}$, Jerônimo C Ruiz ${ }^{5}$, José F Silveira ${ }^{2}$, Patrícia S Cisalpino ${ }^{1^{*}}$

\begin{abstract}
Background: Paracoccidioides brasiliensis (Eukaryota, Fungi, Ascomycota) is a thermodimorphic fungus, the etiological agent of paracoccidioidomycosis, the most important systemic mycoses in Latin America. Three isolates corresponding to distinct phylogenetic lineages of the Paracoccidioides species complex had their genomes sequenced. In this study the identification and characterization of class II transposable elements in the genomes of these fungi was carried out.

Results: A genomic survey for DNA transposons in the sequence assemblies of Paracoccidioides, a genus recently proposed to encompass species $P$. brasiliensis (harboring phylogenetic lineages S1, PS2, PS3) and P. lutzii (Pb01-like isolates), has been completed. Eight new Tc1/mariner families, referred to as Trem (Transposable element mariner), labeled A through $\mathrm{H}$ were identified. Elements from each family have $65-80 \%$ sequence similarity with other Tc1/ mariner elements. They are flanked by 2-bp TA target site duplications and different termini. Encoded DDDtransposases, some of which have complete ORFs, indicated that they could be functionally active. The distribution of Trem elements varied between the genomic sequences characterized as belonging to $P$. brasiliensis (S1 and PS2) and $P$. lutzii. TremC and $\mathrm{H}$ elements would have been present in a hypothetical ancestor common to $P$. brasiliensis and $P$. lutzii, while TremA, B and $F$ elements were either acquired by $P$. brasiliensis or lost by $P$. lutzii after speciation. Although TremD and TremE share about 70\% similarity, they are specific to $P$. brasiliensis and $P$. Iutzii, respectively. This suggests that these elements could either have been present in a hypothetical common ancestor and have evolved divergently after the split between $P$. brasiliensis and $P$. Lutzii, or have been independently acquired by horizontal transfer.

Conclusions: New families of TC1/mariner DNA transposons in the genomic assemblies of the Paracoccidioides species complex are described. Families were distinguished based on significant BLAST identities between transposases and/or TIRs. The expansion of Trem in a putative ancestor common to the species $P$. brasiliensis and $P$. lutzii would have given origin to TremC and TremH, while other elements could have been acquired or lost after speciation had occurred. The results may contribute to our understanding of the organization and architecture of genomes in the genus Paracoccidioides.
\end{abstract}

\footnotetext{
* Correspondence: pscisalp@icb.ufmg.br

'Departamento de Microbiologia, Instituto de Ciências Biológicas,

Universidade Federal de Minas Gerais, 31270-901 Belo Horizonte, MG, Brazil
} 


\section{Background}

The thermodimorphic fungus Paracoccidioides brasiliensis is the etiological agent of paracoccidioidomycosis, a systemic endemic disease that affects at least 10 million people in Latin America [1]. Infection probably occurs as a result of inhalation of conidia that subsequently transform into yeast forms within the lung, resulting in asymptomatic infection or infection that can progress to acute (sub-acute) and chronic clinical forms of the disease [2]. Paracoccidioidomycosis is the 8th most common cause of death due to chronic/recurrent infections and parasitic diseases in Brazil [3].

The teleomorph (sexual) stage of this fungus is unknown, but analysis of the large rDNA subunit classified it as an Ascomycete, order Onygenales, family Onygenaceae, phylogenetically close to Ajellomyces, a genus that harbors 2 other human dimorphic fungal pathogens, specifically Histoplasma capsulatum and Blastomyces dermatitidis [4]. Molecular analysis by random amplification of polymorphic DNA (RAPD) [5], restriction fragment length polymorphism (RFLP) [6] and electrophoretic karyotyping showed extensive genetic variability among distinct isolates $[7,8]$.

RAPD, a technique popular for its simplicity, has been useful in determining the existence of genetically distinct $P$. brasiliensis groups, their relationship with geographic distribution, and the similarity among clinical, animal and environmental samples. However, the information has been difficult to compare. Gene polymorphism studies were pioneered by characterization of $\mathrm{PbGP} 43$ polymorphism, a gene encoding the fungus immunodominant antigen, in a sample of 17 isolates [9]. By comparing 2 sequenced PCR fragments from the whole gene (exon 1, intron and exon 2) these authors found 21 informative substitution sites, mostly in exon 2 , which defined 5-6 genotypes. The maximum-likelihood phylogenetic tree generated with these sequences clearly reflected the presence of specific genetic groups in the species. Subsequently, multilocus genealogy studies demonstrated evolutionary lineages identifying the occurrence of cryptic phylogenetic species that were morphologically indistinguishable by following the criteria of genealogical concordance and non-discordance [10-12] which is used to identify the reduction of gene flow between groups of individuals due to geographic or reproductive isolation barriers. The PbGP43 locus, whose characteristic genotypes have recently been reviewed [13], was the most polymorphic and informative in these studies. Matute et al. [10] analyzed the genetic structure of $65 P$. brasiliensis isolates and concluded that they could be grouped into 3 distinct phylogenetic species: $\mathrm{S} 1$ (including isolate Pb18), PS2 (including isolate Pb03) and PS3 (composed exclusively of Colombian isolates). In a study of 21 P. brasiliensis isolates, 14 of which had been included in the above study, Carrero et al. [11] came to a similar conclusion for all the isolates, with the exception of isolate $\mathrm{Pb01}$, which they suggested was a new phylogenetic species in the genus Paracoccidioides. Recently, Teixeira et al. [12], analyzing 88 isolates of the fungus, found that 17 of them were genotypically similar, belonging to the $\mathrm{PbO1}$ like group. They estimated that the S1/PS2/PS3 species clade and the Pb01-like new species, for which the name Paracoccidioides lutzii was proposed, shared a common ancestor approximately 32 million years ago. These studies suggested that the genus Paracoccidioides consists of at least 4 different, previously unrecognized phylogenetic species: P. brasiliensis S1 (species), PS2 (phylogenetic species 2) and PS3 (phylogenetic species 3), and a 4th quite distinct lineage comprising fungal isolates formerly referred to as Pb01-like [11], now proposed to be a new species, $P$. lutzii [12]. The multilocus sequence analysis also supported recombination in nature in $P$. brasiliensis (S1) [10] and P. lutzii [11,12], indicating the presence of sexual reproduction. Thanks to The Broad Institute Fungal Genome Initiative (FGI) Project, the complete genome of representative isolates from $P$. brasiliensis $\mathrm{S} 1$ (Pb18), PS2 (Pb03) and Pb01 like (Pb01) have recently been made available at the site: http:// www.broad.mit.edu/annotation/genome/paracoccidioides_brasiliensis thus allowing further and specific comparisons among isolates. Previous local projects, however, contributed to the construction of large expressed sequence tag databases of $\mathrm{Pb} 18$ [14] and $\mathrm{Pb} 01$ $[15,16]$.

Transposable elements (TEs) represent a substantial fraction of eukaryotic genomes that have been found in virtually all species investigated to date. They are abundant in fungi and represent $3-20 \%$ of the genomes of many filamentous fungi $[17,18]$. TEs can produce a variety of effects in genomes, such as gene inactivation or modification, chromosome breakage, and genome recombination or rearrangement, thereby generating plasticity [19]. Karyotyping and gene mapping indicated the occurrence of chromosomal rearrangements [8], which are associated with the presence of repetition and TE in fungal genomes [17].

TEs can be grouped into 2 classes according to their transposition mechanism. Class I consists of retrotransposons that transpose via an RNA intermediate that is reverse-transcribed into DNA by a TE-encoded reverse transcriptase. Class II elements, also called DNA transposons, move via a DNA intermediate. Because of the abundance and diversity of TEs described in the eukaryotic genomes, a unified classification system for eukaryotic TEs has been proposed by different groups $[20,21]$. 
DNA transposons can be divided into 3 subclasses: the first comprises the elements that transpose via the classical "cut-and-paste" mechanism; the second comprises elements that transpose by replication involving the displacement of only one strand; and in the third, transposons move by a mechanism called "rolling-circle" [20,21].

Subclass 1 DNA transposons require the cleavage of both DNA strands for the transposition and have 2 orders - TIR and Crypton. Six superfamilies of the order TIR from subclass 1 have been identified in fungi: Tc1/ mariner, hAT, Mutator, Transib, PIF-Harbinger and CACTA. TIR transposases contain a signature active site motif that consists of 3 acidic amino-acids (DDE or DDD) and forms the catalytic pocket of the enzyme responsible for cleaving DNA strands. Crypton, the only superfamily representative of the second order, has only been found to date in fungi and, unlike any previously described transposons, encodes a putative tyrosine recombinase instead of a classical transposase. This recombinase lacks an $\mathrm{RT}$ domain, which suggests that transposition occurs via a DNA intermediate. It does not contain long repeat sequences, but is bordered by short direct repeats that may have been generated by its insertion into the host genome by recombination. Cryptons may be excised from the host genome by tyrosine recombinase, generating a circular, extrachromosomal, double-stranded DNA molecule, for which cleavage of both DNA strands is required. Elements from subclasses 2 (Helitrons) and 3 (Maverick) have only one strand cleaved during transposition and have also been identified in fungi, but the Tc1/mariner superfamily is probably the most prevalent in these organisms [20-22].

Transposons contain terminal inverted repeats (TIRs), which are recognized by the pipsqueak helix-turn-helix (HTH) motif in the transposase domain. Each subclass 1 DNA transposon superfamily is characterized by a specific transposase core that is different from those of other superfamilies. Upon insertion, DNA transposons generate target site duplications (TSD) with lengths that are relatively well conserved among superfamily members [20,21].

TEs have been poorly characterized in P. brasiliensis. Transcriptome analysis indicated that Ty-like retroelements were among the most abundant ESTs in P. brasiliensis [15]. Nascimento et al. [23], searching for microsatellites that could be potentially useful as molecular markers, reported that a small number of them were located in transposons. The focus of our work has been the characterization of DNA transposons and their derivatives in the genus Paracoccidioides. To this end we searched for DNA transposons in the Paracoccidioides genomic sequence database http://www.broad. mit.edu/annotation/genome/ paracoccidioides_brasiliensis. We have discovered 7 new families of DNA transposons and one new subfamily of Mariner-1_AF belonging to the Tc1/mariner superfamily. Remarkably, some Trem (Transposable element mariner) elements appear that may be active in the genomes of representative isolates of 2 different Paracoccidioides phylogenetic lineages. The finding of active autonomous DNA transposons in P. brasiliensis may have implications for an understanding of the evolutionary processes underlying the diversification of this group. Furthermore, transposons are efficient vectors for introducing foreign DNA into cells.

\section{Results}

\section{Occurrence of DNA transposons in the genus} Paracoccidioides

A survey of the P. brasiliensis Pb01 Functional and Differential Genome Project annotated EST database (PbDBEST) [24] for sequences similar to transposase sequences found 2 contigs (530 and 1938, with 673 and 637 bp respectively) that contain open reading frames (ORF) encoding proteins of 155 and 71 amino acids, respectively, with similarity to the conserved HTH DNA binding domain of the transposase of the Aft 1 DNA transposon in Aspergillus fumigatus. These sequences were amplified from $P$. brasiliensis genomic DNA, their identities confirmed by sequence analysis, and then used as probes in Southern blot hybridization with genomic DNA of different $P$. brasiliensis isolates. This revealed the presence of multiple copies of DNA transposons dispersed throughout the genome of different isolates. RT-PCR analysis of total RNA of the isolates $\mathrm{Pb} 01$ (recently proposed to be a new species, P. lutzii) [12], Pb03 and Pb18 (P. brasiliensis, corresponding to distinct phylogenetic lineages known, respectively, as S1 and PS2) [10] confirmed transcription of the putative elements (data not shown).

The transposon sequence from each EST contig was used as a query in a modified BLASTN search (see Methods section for details) against the whole genome sequence assemblies of all 3 Paracoccidioides genomes (Pb01, Pb03 and Pb18) and combined with the strategy for identification of TIRs, what allowed the Artemis annotation tool to be used to identify transposons.

Table 1 shows the number of sequence copies with similarity to transposases identified in the genome of each isolate sequenced. In total, 1332 hits were unambiguously identified in the genomes of Pb01 ( $\mathrm{n}=384)$, $\mathrm{Pb} 03(\mathrm{n}=475)$ and Pb18 $(\mathrm{n}=473)$. They were predicted to have features that allowed them to be classified in the Tc1/mariner superfamily. A significant number of the hits found ( $\mathrm{n}=868 ; 65.2 \%$ ) corresponded to highly defective elements, with lower conservation of the typical transposase domains (HTH, CENPB and/or DDE), which have still to be analyzed for classification (Table 1). 
Table 1 Abundance of DNA transposons identified and classified in the different isolates of $P$. brasiliensis ${ }^{a}$

\begin{tabular}{ccccc}
\hline & Pb01 & Pb03 & Pb18 & Total \\
\hline Classified $^{\mathrm{b}}$ & $102(26.6 \%)$ & $177(37.3 \%)$ & $185(39.1 \%)$ & $464(34.8 \%)$ \\
Not classified $^{\mathrm{c}}$ & $282(73.4 \%)$ & $298(62.7 \%)$ & $288(60.9 \%)$ & $868(65.2 \%)$ \\
Total & $384(100 \%)$ & $475(100 \%)$ & $473(100 \%)$ & $1332(100 \%)$ \\
\hline
\end{tabular}

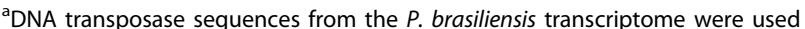
to perform queries in a modified BLASTN search (see Methods) in the genome of isolates $\mathrm{Pb} 01, \mathrm{PbO} 3$ and $\mathrm{Pb} 18$ and combined with a strategy for identification of TIRs.

bElements assigned to a DNA transposon superfamily according to the standard principles of the TE classification proposed by Wicker et al. [20].

'Sequences that do not meet all the criteria proposed by Wicker et al. [20] They are highly defective elements, with lower conservation of the typical transposase domains (HTH, CENPB and/or DDE).

We also searched the Paracoccidioides genomes for Cryptons, rolling-circle DNA transposons (Helitrons) and self-synthesizing DNA transposons (Polintons) $[20,21]$, using consensus sequences for these elements (tyrosine recombinase, helicase, replication protein $\mathrm{A}$ and DNA polymerase B), no significant matches were found.

In the genomic sequence of Pb01 ( $P$. lutzii), only 3 DNA transposon families have been classified, corresponding to $102(26.6 \%)$ of the 384 hits found in this isolate (Table 1). In the set of elements for Pb03, 6 fulllength potentially functional autonomous elements coding for putative transposases with well-defined TIRs were classified. The copies of the 6 elements found in Pb03 totaled 177 hits, or $37.3 \%$ of the 475 hits, similar to DNA transposons in this isolate (Table 1). Of the 473 hits registered in Pb18, 185 (39.1\%) were identified and correlated with Tc1/mariner elements.

\section{Tc1/mariner superfamily in the genus Paracoccidioides}

We identified 8 DNA transposon elements in Paracoccidioides that share $65-80 \%$ identity at the nucleotide level with Tc1/mariner transposons deposited in Repbase [25] (details in Additional file 1) at the Genetic Information Research Institute (GIRI). Like the Tc1/mariner transposons, 6 of these elements are flanked by TA dinucleotide (Figure 1). Because of their similarity to the Tc1/mariner superfamily and the fact that most of them (61.6\%) insert specifically in TA target sites, these elements were given the name Trem (an abbreviation for Transposable element mariner). The completeness of transposons was confirmed by the presence of TIRs at both ends, followed by multiple sequence alignment to well-studied examples (Table 2, Figure 2). Incomplete sequences were not included in the comparative analyses shown in Figure 2. We examined 464 insertion loci of Trem elements (Table 2 ), of which 321 (69.2\%) are flanked by well-defined TIRs, while the remaining sequences, including all 17 TremG elements, are not flanked by TIRs.

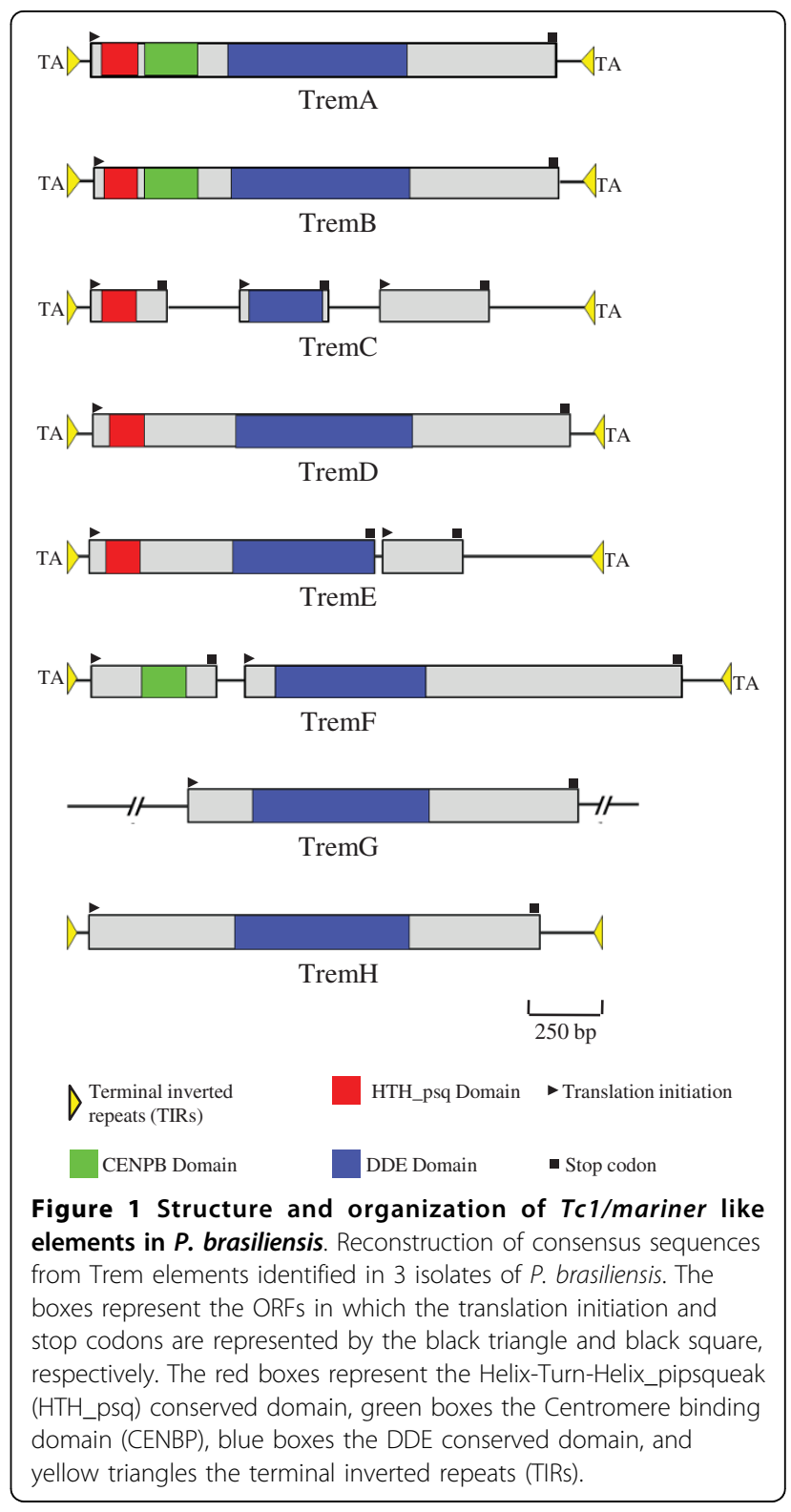

There are 7 copies of TremA in the Pb03 isolate, 5 which contain a full-length ORF and 2 which are truncated, and 11 copies in the Pb18 isolate, 5 which have an intact ORF (Figure 1, Table 2). TremA sequences were aligned to build an $1850 \mathrm{bp}$ consensus containing a single ORF (nt 85-1728) that encodes a 547 amino acid protein with 3 conserved domains characteristic of transposases - HTH, CENPB and DDE (Figure 1). The TremA TIRs are $49 \mathrm{bp}$ long and have a $17 \mathrm{bp}$ internal direct repeat (Figure 2). Analysis of TremA with the RepeatMasker script in the Girinst database http://www. girinst.org/repbase revealed a similarity of only $66 \%$ with the A. fumigatus Mariner-1_AF transposon, suggesting that it may be a new DNA transposon family [20]. 
Table 2 Characteristics of the Tc1/mariner transposons identified in the genome of $P$. brasiliensis.

\begin{tabular}{|c|c|c|c|c|c|c|c|c|c|}
\hline \multirow[t]{2}{*}{ Transposon } & \multirow[t]{2}{*}{$\mathrm{TSD}^{\mathrm{a}}$} & \multirow[t]{2}{*}{ Repeat Masking $^{\mathbf{b}}$} & \multirow[t]{2}{*}{ Size $^{c}(b p)$} & \multirow[t]{2}{*}{$\operatorname{Protein}^{\mathrm{d}}$ (aa) } & \multirow[t]{2}{*}{$\operatorname{TIRs}^{\mathrm{e}}(\mathrm{bp})$} & \multicolumn{4}{|c|}{ Copy number ${ }^{f}$} \\
\hline & & & & & & $\mathrm{Pb} 01$ & $\mathrm{Pb03}$ & $\mathrm{Pb} 18$ & $\mathrm{~N}^{\mathrm{g}}$ \\
\hline TremA & TA & 66\% (Mariner-1_AF) & 1850 & 547 & 49 & - & $7(5)$ & $11(5)$ & 18 \\
\hline TremB & TA & 65\% (Mariner-1_AF) & 1857 & 544 & 45 & - & $15(2)$ & 21 & 36 \\
\hline TremC & TA & $66 \%$ (Mariner-2_AO) & 1869 & $95,103,127$ & 49 & 48 & 67 & 50 & 165 \\
\hline TremD & TA & 80\% (Mariner-1_AF) & 1891 & 559 & 45 & - & - & $5(4)$ & 5 \\
\hline TremE & TA & 65\% (Mariner-1_AF) & 1883 & 335,217 & 42 & 25 & - & - & 25 \\
\hline TremF & TA & 67\% (Mariner-9_An) & 2329 & 143,505 & 43 & - & 12 & 25 & 37 \\
\hline TremG & - & 66\% (Mariner-5_AF) & - & 460 & - & - & 6 & 11 & 17 \\
\hline TremH & - & 66\% (Mariner-3_AO) & 1882 & 525 & 42 & 29 & 70 & 62 & 161 \\
\hline Total & & & & & & 102 & 177 & 185 & 464 \\
\hline
\end{tabular}

${ }^{\mathrm{a}}$ TSD - Target Site Duplication. TA dinucleotide.

${ }^{b}$ Repeat Masking - results of the analysis performed with the Repeat Masking script using the Girinst http://www.girinst.org/repbase database and percentage similarity.

'Size - total length of element in base pairs (bp)

${ }^{d}$ Protein - Length of transposase in amino acids (aa). Some elements contain 2 or 3 ORFs encoding different proteins (see Figure 1).

'TIRs - Length of Terminal Inverted Repeats in base pairs (bp).

${ }^{f}$ Copy number - Number of TE copies found in the genome of the $3 P$. brasiliensis isolates. The number of elements with potentially functional ORFs is shown in parentheses.

${ }^{9} \mathrm{~N}$ - The total number of $T c 1 /$ mariner transposons identified in $P$. brasiliensis.

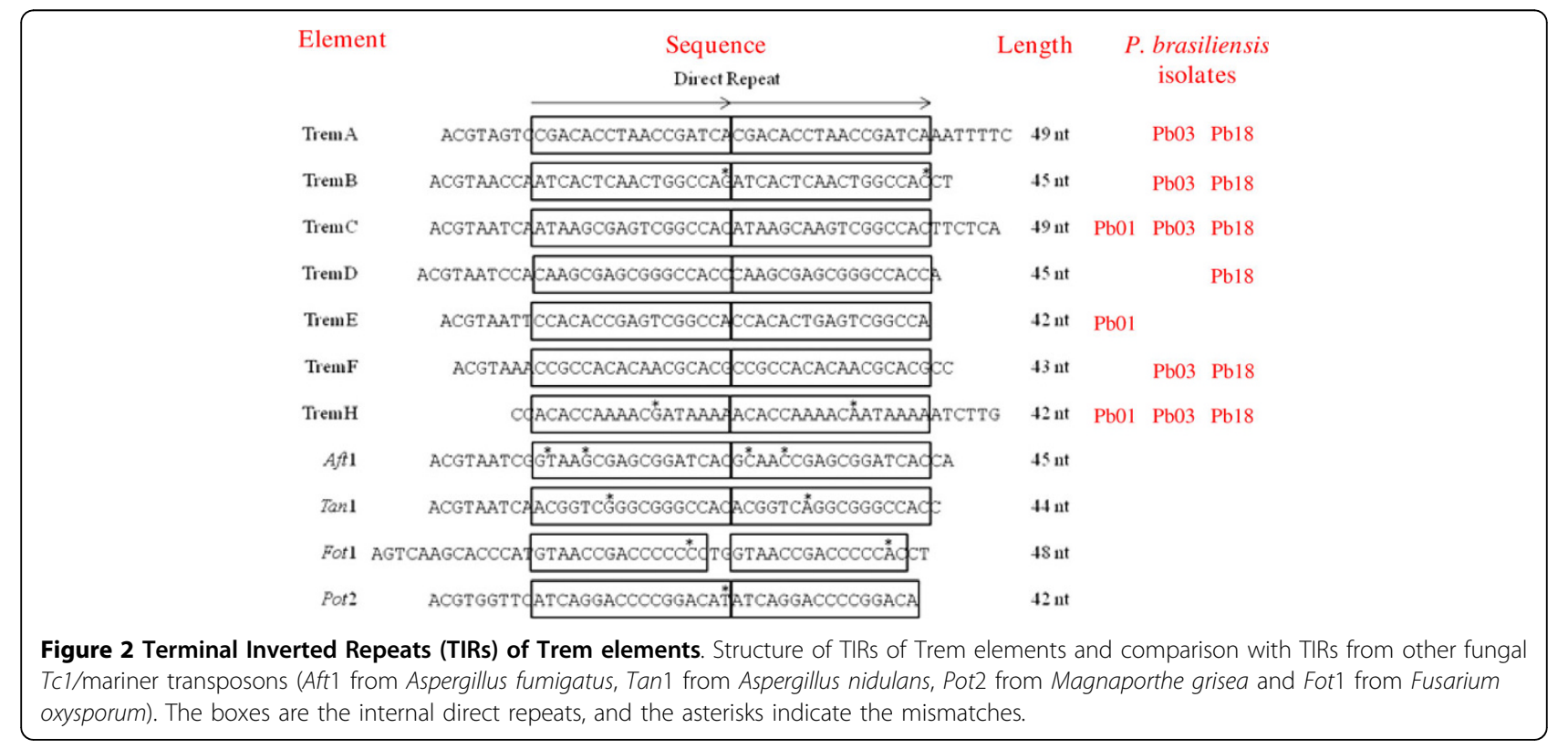

We found 36 copies of TremB, 15 in the genome of the $\mathrm{Pb} 03$ isolate and 21 in Pb18 (Table 2). However, only 2 copies showed intact ORFs, which were used to generate a $1857 \mathrm{bp}$ consensus sequence flanked by $45 \mathrm{bp}$ TIRs (Table 2, Figures 1 and 2). This ORF (nt 130-1764) encodes a 544 aa transposase with 3 conserved domains ( $\mathrm{HTH}, \mathrm{CENPB}$ and DDE; Figure 1). Analysis using RepeatMasker gave an index of $65 \%$ similarity with the Mariner-1_AF. It is interesting that 25 TremB sequences carrying truncated ORFs are flanked by intact TIRs.

TremC is the most abundant DNA transposon, having 165 truncated copies dispersed throughout the genomes.
The 32 longest sequences were aligned to generate a $1869 \mathrm{bp}$ consensus flanked by 49 bp TIRs (Table 2, Figures 1 and 2). It contains 3 short ORFs, 2 which encode the HTH and DDE domains (Figure 1). The central region of the element shows similarity with $A$. fumigatus transposases, and analysis by RepeatMasker revealed 66\% similarity with Mariner-2_AO in Aspergillus oryzae. The TremC shown in Figure 1 is based on the element's most conserved sequences.

TremD is present in a small number of copies $(n=5)$, exclusively in the Pb18 isolate (Table 2). The consensus sequence has $1891 \mathrm{bp}$ and harbors a $1680 \mathrm{bp}$ ORF 
(nt 95-1774) flanked by 45 bp TIRs (Figures 1 and 2). The ORF codes for a 559 aa transposase carrying the $\mathrm{HTH}$ and DDE domains (Figure 1). RepeatMasker analysis showed $68 \%$ similarity with Mariner-1_AF in the central region, and $80 \%$ similarity with the TIRs, suggesting that TremD is related to or belongs to this family [20].

The consensus sequence for the TremE transposon, found exclusively in the Pb01 isolate, is $1883 \mathrm{bp}$ long, flanked by 42 bp TIRs (Table 2, Figures 1 and 2). It includes 2 ORFs: one has 1008 bp (nt 78-1085) and codes for a 335 aa transposase with the HTH and DDE domains; and the second (654 bp long, nt 1113-1766) encodes a 217 aa protein similar to a hypothetical protein (1e-47) from Ajellomyces capsulatum (Figure 1). TremE showed a $65 \%$ similarity with the Mariner-1_AF transposon.

With its 2329 bp consensus sequence, TremF is the longest of the elements described in this study and the third most abundant $(\mathrm{n}=37)$ (Table 2, Figure 1$)$. The consensus sequence is flanked by 43 bp TIRs and contains 2 ORFs: the first is 432 bp long (nt 89-520) and codes for a 143 aa protein harboring the conserved domain CENPB; the second extends from position nt 650 to 2167 (1518 bp) and codes for a 505 aa DDE-domain-harboring protein (Figures 1 and 2). The results of RepeatMasker analysis revealed a $67 \%$ similarity with Mariner-9_AN in Emericella nidulans, which is a non-autonomous DNA transposon belonging to the pogo clade. Thus, TremF could represent a new transposon family [20].

TremG, the 7 th Tc1/mariner element we identified, has 6 copies in Pb03 and 11 in Pb18 (Figure 1, Table 2). All copies are truncated, flanked by highly divergent and irregular sequences (35-124 bp). The central core of some elements has an ORF coding for a 460 aa protein carrying the DDE domain. Unfortunately, it was not possible to determine the precise boundary of this element because of the divergence among the flanking sequences. RepeatMasker analysis showed a $66 \%$ similarity with the Mariner-5_AF in A fumigatus, a DNA transposon of the Tc1 clade. For this reason, this element was classified as a Tc1/mariner transposon.

TremH is present in the 3 isolates, with 29 copies in $\mathrm{Pb} 01,70$ in Pb03 and 62 in Pb18 (Figure 1, Table 2). Of the 161 copies of this element, 120 (74.5\%) are flanked by $42 \mathrm{bp}$ TIRs; however, it was not possible to define its target site. We used 6 copies that had the best sequence conservation (94\% identity among them) and encoded the DDE motif to rebuild the 1882 bp consensus sequence (including two $42 \mathrm{bp}$ flanking TIRs). RepeatMasker analysis showed a $66 \%$ similarity with the Mariner3_AO in $A$.oryzae. The element copy contains an ORF coding for a 525 aa protein carrying the DDE motif (Figure 1), similar to that in the transposon in Talaromyces stipitatus (2e-102) (Table 2). In this region,
3 strictly conserved acidic amino acids - D(176), D(289) and $\mathrm{D}(325)$ (positions relative to the transposase sequence) - were found, and these probably constitute the DDE catalytic motif in TremH. Since the transposase activity in many DNA transposons relies upon the catalytic activity of the DDE domain, we suggest that TremH is a Tc1/mariner-like transposon.

\section{Phylogenetic analysis}

The alignment of the predicted sequences corresponding to each of the conserved transposase domains found in 8 DNA transposons in Paracoccidioides, with the sequences of well-characterized DNA transposons of the Tc1/mariner superfamily (Figure 3) from other species, allows the identification of motifs considered essential for active transposition (Figure 3). Some of the Paracoccidioides transposases are interrupted by stop codons, minor indels or the absence of a translation initiation codon (Table 2, Figure 1 and Additional file 2). The complete catalytic triad DDE occurred in 6 of the elements (TremA, B, D, E, G and H) and was characterized by the substitution of the glutamate residue by aspartate to form a D, D(35 residues), D motif, as previously observed in other fungal transposases. The triad signatures (the triad residues and the distances between them) are very similar and can be represented by D10(111 residues)-D122-(35 residues)-D158 in the elements TremA, B, D and E; D10-(109 residues)-D120-(35 residues)-D156 in the element TremG; and D7-(111 residues)-D119-(35 residues)-D155 in the element TremH. In TremF, the first aspartic residue was deleted, but the distance between the second and third aspartate residues was maintained (-D89-(35 residues)-D125). In TremC, all 3 aspartic residues were absent.

In addition to the conserved catalytic triad DDD, transposases contain a number of other highly conserved, group-specific amino acids, such as the $\mathrm{HTH}$ and CENPB domains. The HTH domain was found in 5 Trem elements (TremA, B, C, D and E) with varying degrees of conservation - $58 \%$ identity between the pairs TremA/TremB, TremB/TremC and TremD/TremE, and $53 \%$ identity between TremA/TremC. The identities between TremE/TremA and TremE/TremB were lower than $40 \%$. Alignment of the DNA-binding domains of the transposases revealed a distinction between Trem elements, which may be functionally connected to the TIRs of these elements.

When the HTH domain was compared with that of other fungal transposases, the highest identity index (60\%) was found between TremD and the Tan 1 transposon of Aspergillus niger. The CENPB domain was present in 3 of the Paracoccidioides transposons (TremA, B and F). The identity between TremA and TremB was $65.6 \%$, and between these elements and Trem F less than $36 \%$. 


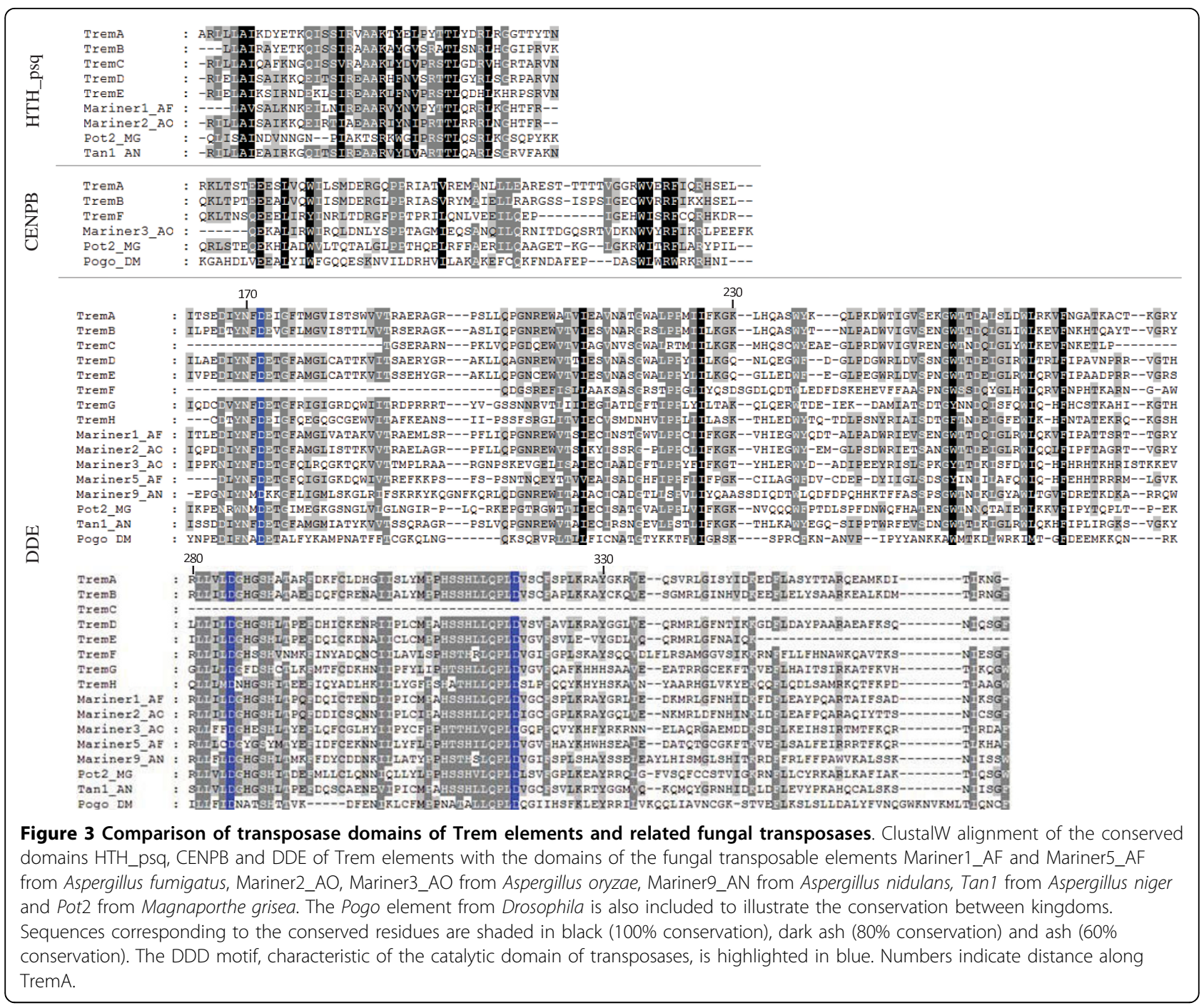

It was also observed that 3 elements (TremA, TremB and TremD) presented conserved $\mathrm{Cx} 2 \mathrm{Cx} 4 \mathrm{Hx} 4 \mathrm{C}$ zinc finger domains in the $\mathrm{C}$-terminal ends of their transposases corresponding, respectively, to amino acid residues 531-544, 528-541 and 540-553. In TremC and TremE, similar but non-canonical motifs were present at the Cterminal regions corresponding, respectively, to the residues 290-315 (CCx18Cx4H) and residues 404-419 (Cx2Cx2Hx8G).

Phylogenetic analysis using amino acid sequences clustered the transposases of Paracoccidioides into 2 major groups, one carrying TremA, B, C, D, E and F elements, and the other harboring TremH and $\mathrm{G}$ elements (Figure 4). This analysis confirms the proximity between TremA, TremB and TremC elements (95\% bootstrap) and TremD and TremE elements (99\% bootstrap). TremF elements, although clustered with the Trem elements above, are divergent and located in a distinct branch. Based on phylogenetic reconstructions (Figure 4), TremG and
TremH seem to form a cluster completely separated from other Trem elements. Both groups contain Tc1/ mariner transposases that are also present in other fungi, e.g. A. nidulans, A. fumigatus and A. oryzae.

Our analysis suggests that Trem elements of the different Paracoccidioides genomes were derived from a single ancestral sequence present before they diverged from one another. At a later point in the evolution of this fungus, transposases split into 2 separate lineages, giving rise to the family composed of TremA, B, C, D, E and $\mathrm{F}$ and the other consisting of TremH and $\mathrm{G}$.

\section{Distribution and genomic environment of Trem elements}

To characterize the insertion target sites and preferences in Paracoccidioides genomes in more detail, junction fragments between the transposon and the genome were analyzed. Trem elements, like other Tc1/mariner transposons, integrate into a TA dinucleotide, causing targetsite duplication of the TA sequence at the TIR 


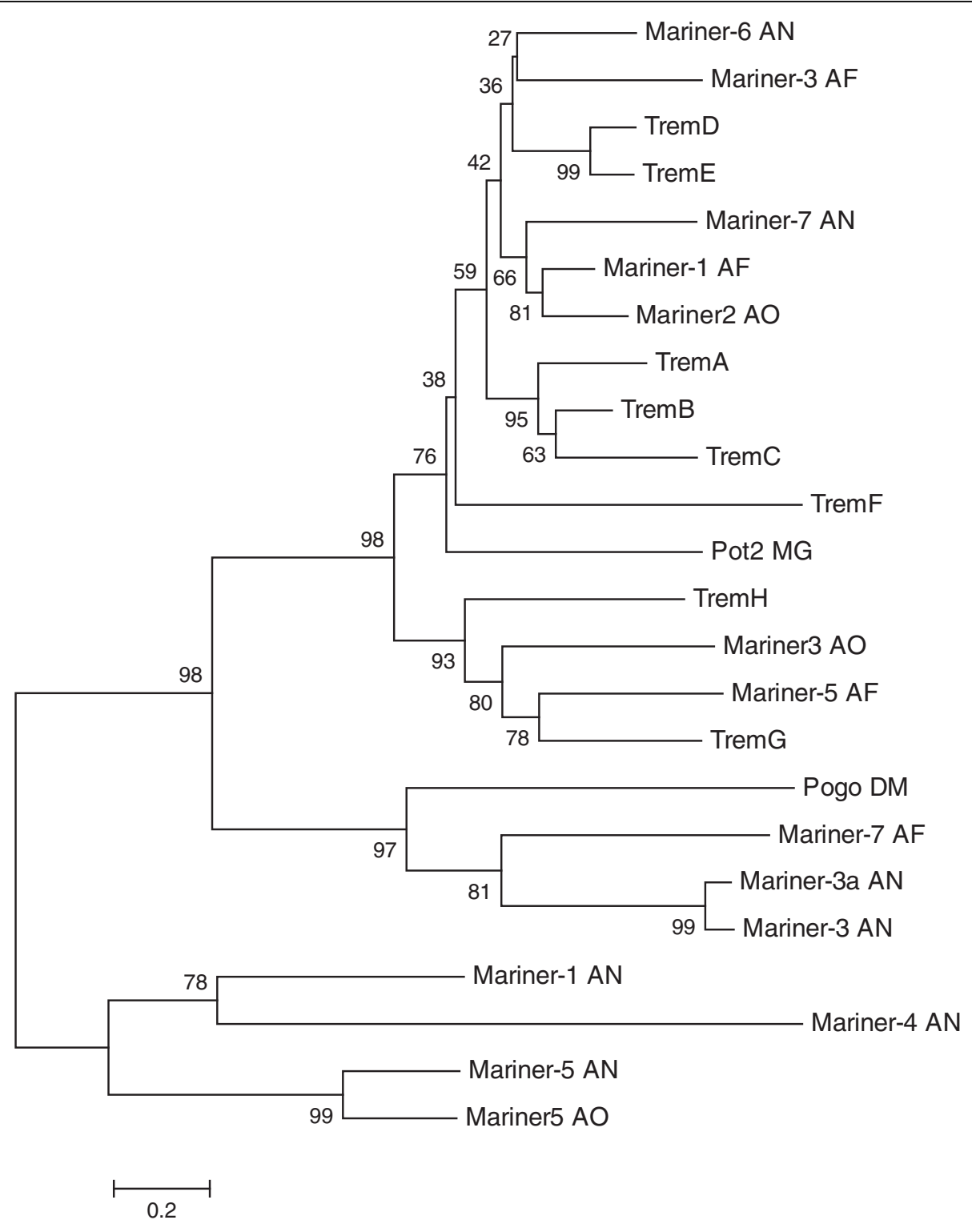

Figure 4 Phylogeny of Trem elements and related fungal transposons. Phylogenetic tree of the predicted amino acid sequences of Trem elements of $P$. brasiliensis and different $T c 1 /$ mariner transposons. The list of the sequences used to construct alignments and phylogenetic trees is given in the Methods section. Neighbor-joining trees were constructed using the equal-input model with 5,000 bootstrap replicates. Numbers near the individual nodes denote BioNJ bootstrap values. The scale bar corresponds to 0.2 substitutions per site.

boundary. Additional file 2 lists 321 independent insertion events identified in the 3 Paracoccidioides genomes, demonstrating integration of the transposon into a TA dinucleotide within the fungal genome and validating genuine transposition.

Analysis of the genomic location of each Trem element insertion showed that they were dispersed across the genomes. They did not, however, appear to be completely random (additional file 3). Genome sequences from isolate $\mathrm{Pb} 03(29.05 \mathrm{Mb})$ were assembled in 65 supercontigs (scaffolds). The 475 hits similar to DNA transposons (Table 1) were found along 53 of the 65 supercontigs of Pb03. Of these 53, 39 contain Trem elements $(\mathrm{n}=177)$, which account for $1.14 \%$ of the genome of isolate $\mathrm{Pb} 03$ (additional file 4). In the genome of Pb18 (29.95 Mb), there are 473 hits similar to DNA transposons (Table 1) distributed in 44 of the 57 supercontigs, and Trem elements $(n=185)$ were found in 36 supercontigs of this isolate (additional file 5), covering $1.11 \%$ of the genome. In isolate $\mathrm{Pb} 01$ (genome size $32.94 \mathrm{Mb}, 111$ supercontigs), the 384 hits showing similarity to TEs (Table 1) were distributed along 87 
supercontigs (approximately one element each $85 \mathrm{~kb}$ ), and Trem elements accounted for $0.58 \%$ of the genome (additional file 6).

The above results suggest that Trem elements do not integrate in a completely random fashion. In an extended survey, the genomic context in which Trem elements were inserted was examined in $100 \mathrm{~kb}$ region of supercontigs 1 (length $3.65 \mathrm{Mb}$ ) and 18 (length 304 $\mathrm{kb}$ ) of isolate Pb03 (Figure 5). In supercontig 1, this particular region has a high density of DNA transposons (7 hits), 6 of which belong to the Trem family (3 TremC, 2 TremH and 1 TremB) (Figure 5). In addition to the 6 Trem elements, 10 genes (including those coding for thioesterase, lanthionine synthetase and hypothetical proteins) and 12 pseudogenes are present. Among the pseudogenes there is an unclassified pogo-like putative DNA transposon. In supercontig 18, we found 5 Trem elements ( 2 TremH, 1 TremA, 1 TremB and 1 TremC), 3 genes (cAMP kinase dependent protein, MSH2 and hypothetical protein) and 17 pseudogenes. The analysis showed that Trem elements target a wide variety of chromosomal positions and some appearing to be clustered in several regions of the genome.

\section{Southern blot and chromoblot hybridization analyses}

Southern blot hybridization of Paracoccidioides genomic DNA with TremA, TremB and TremE probes revealed multiple bands, suggesting that multiple copies were inserted with polymorphic distribution (Figure 6A). Differences between the hybridization profiles obtained with the same Trem probe confirm the existence of different copy numbers and distribution of the element in the isolates. No hybridization signal was observed in isolate $\mathrm{Pb01}$ (P. lutzii) with probes TremA or TremB. TremE probe hybridized with 10 well-defined bands in $\mathrm{Pb} 01$ isolate, and weakly with a $1.0 \mathrm{~kb}$ band in Pb18 isolate ( $P$. brasiliensis; Figure 6A). Probe TremA hybridized with 3 of the 4 chromosomal bands observed in $\mathrm{Pb} 03$ and with 4 of the 5 in Pb18. Chromoblot hybridization also confirmed the absence of TremA and TremB in isolate Pb01 (Figure 6B). The P. brasiliensis synteny map shows the location of 17 scaffolds in the 5 chromosomes of isolate $\mathrm{Pb} 18$ : http://www.broadinstitute.org/annotation/genome/paracoccidioides_brasiliensis/Chromomap. html. TremA and TremB elements are present in 4 chromosomes, but are not found in chromosome 5, in accordance with their karyotype profiles (Figure 6B).

\section{PCR amplification of Trem elements in Paracoccidioides isolates of distinct phylogenetic origin}

TIRs of Trem elements consist of two 17 bp direct repeats similar to those found in DNA transposons of other fungi, namely $A f t 1$ and Tan 1 (Figure 2). The repeats are completely conserved except for the TIRs of the elements TremB and TremH, in which one substitution was detected (Figure 2). PCR screening using primers derived from TIRs of each Trem element was performed on genomic DNA from 17 clinical isolates, including those from the FGI genome project, and one environmental isolate (Table 3).

Of the 18 isolates, 15 had been previously genotyped by sequencing and their phylogenetic relatedness was known; the phylogenetic relatedness of the remaining 3 isolates - 2 Brazilian (Ed01, 1578) and one Argentinean (utero) which had not been included in previous sequencing and multi-locus studies - was unknown (Table 3). Previous work using RAPD for typing P. brasiliensis indicated that the employment of certain primers and conditions allowed discrimination of isolates with very low genetic similarity and clustering of the most similar ones $[5,6,26,27]$. We considered that performing RAPD with these highly discriminatory primers would allow us to build a dendrogram in which the genetically most distant branches would correspond to the species level, P. brasiliensis and P. lutzii (Pb01-like), clustering isolates corresponding to the phylogenetic species S1 and PS2, an assumption that would uncover the phylogenetic relatedness of the 3 unknown isolates (Ed01, 1578 and utero). The 18 isolates submitted to RAPD analysis were classified as belonging to 3 of the 4 known phylogenetic lineages: S1 and PS2 (P. brasiliensis) or Pb01-like (P.lutzii) [10-12] (Figure 7). Analysis of the dendrogram showed 2 major clusters with a low coefficient of similarity (approximately 6\%), the first corresponding to P. lutzii (Pb01, Ed01 and 1578). The second branch comprised all $15 P$. brasiliensis isolates showing 2 internal clusters (80\% similarity), the first including 10 $\mathrm{S} 1$ and the second clustering 3 PS2 isolates $(\mathrm{Pb} 2, \mathrm{~Pb} 03$ and $\mathrm{Pb} 4$; Table 3) [10-12]. The P. brasiliensis isolates Penguin and $\mathrm{Pb} 12$, although previously defined as S1 (Table 3), did not group with the $\mathrm{S} 1$ isolates but appear as 2 branches each with only one strain. It is interesting that the isolates Penguin and Pb12 are from Uruguay and Argentina, while the majority of the other isolates are Brazilian (13, one Venezuelan - Pb2- and one Peruvian - Pb10). A fungal associated feature that has been reported to correlate by RAPD branching or clustering of Paracoccidioides isolates refers to the isolate's geographic origin [6].

PCR analysis performed with primers corresponding to the TIRs produced amplicons with sizes compatible with the Trem elements described above, confirming the results obtained in the genomic analysis (Figure 8). To confirm the PCR specificity, the amplicons were hybridized with probes containing internal regions of each Trem element (Figure 8). As expected, TremA was amplified in $\mathrm{Pb} 03$ and $\mathrm{Pb} 18$ (P. brasiliensis, phylogenetic species PS2 and S1, respectively) as well as in other 


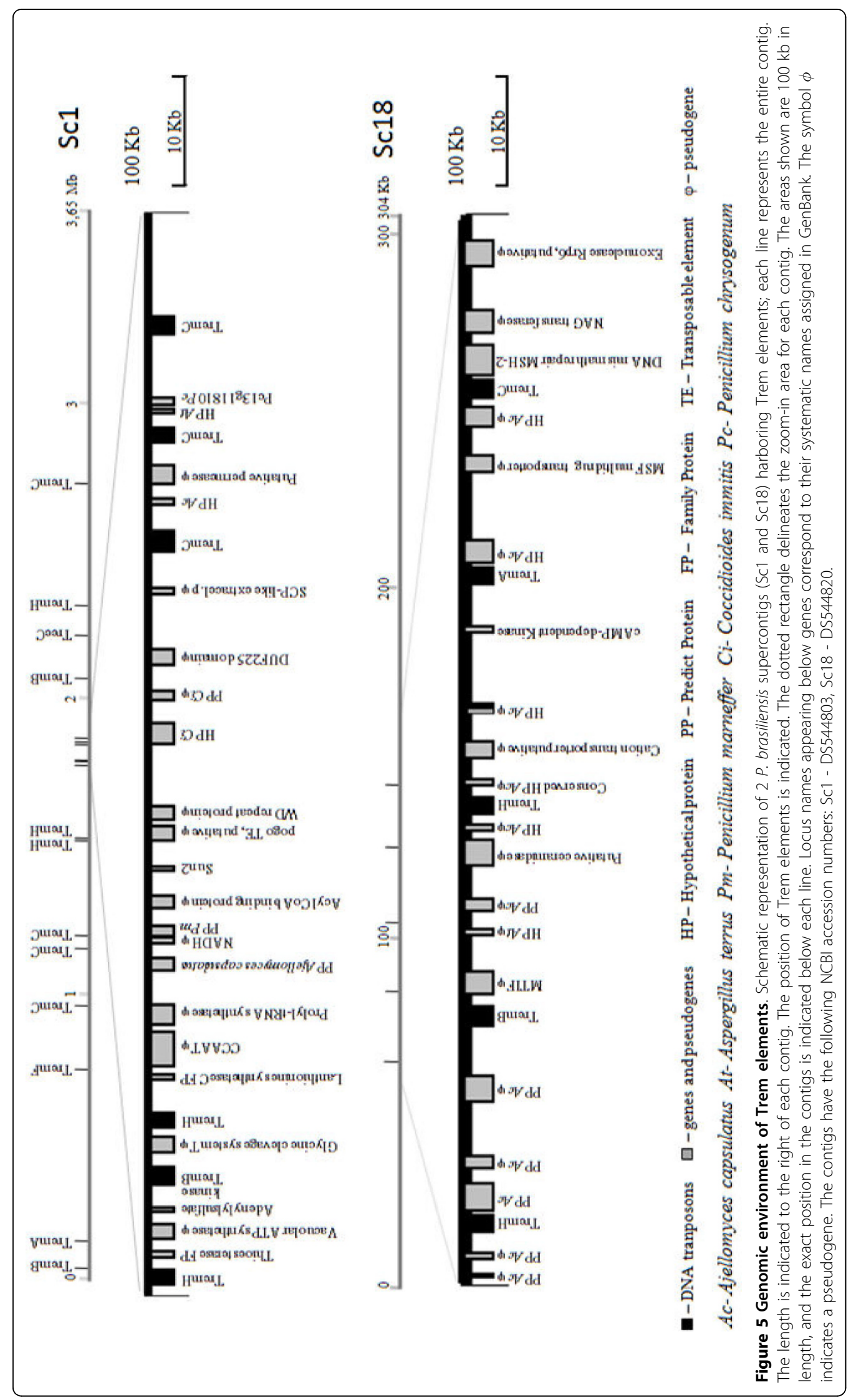




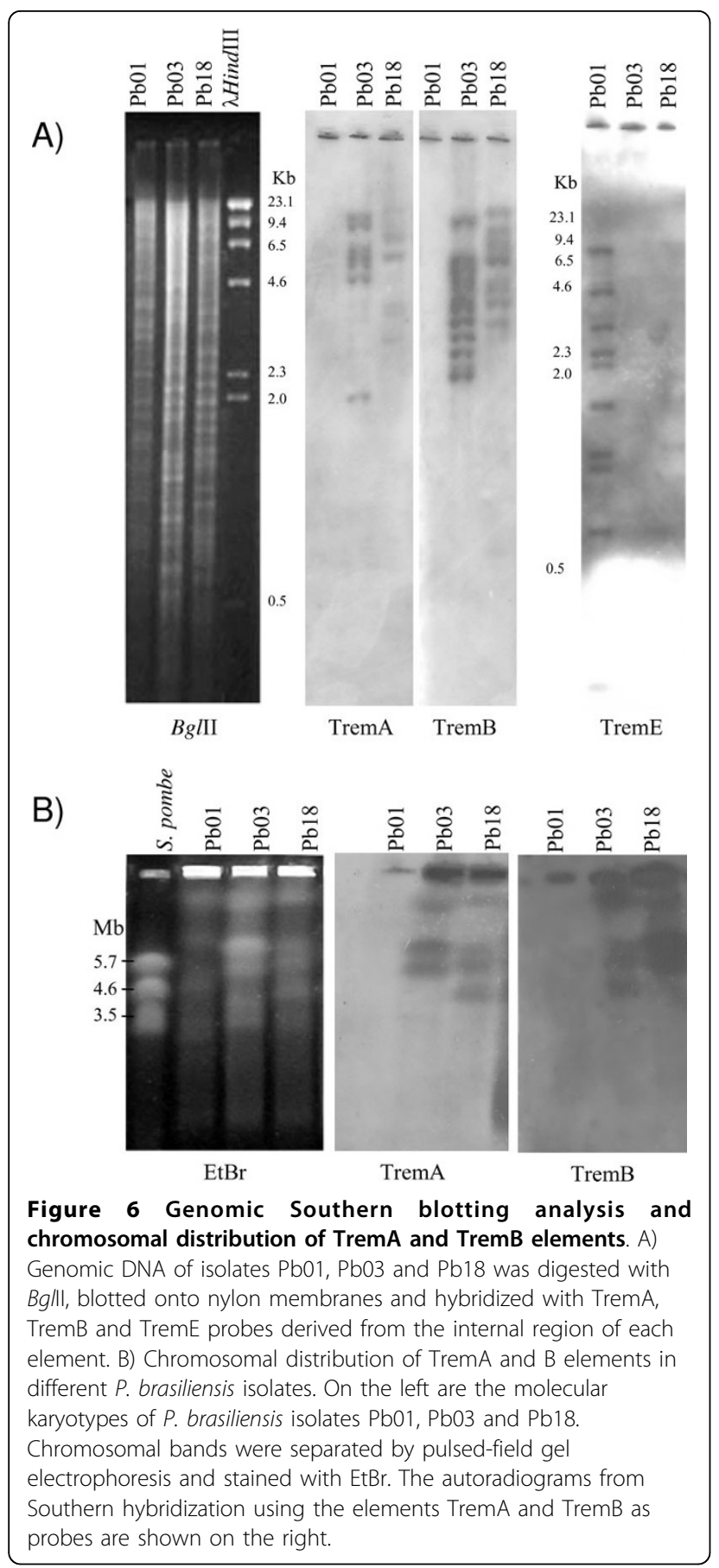

isolates phylogenetically related to them, except for Penguin and Pb06 isolates. A similar amplification pattern was found for TremB in all PS2 and S1 isolates, except for isolate $\mathrm{Pb} 14$. TremA and TremB were not found in Pb01-like isolates. TremC was found in all isolates. A strong hybridizing $1.9 \mathrm{~kb}$ band was detected in the Pb01-like isolates. Two bands (1.9 and $1.8 \mathrm{~kb})$ were amplified in the PS2 and S1 isolates; however, only the largest ones hybridized to the TremC probe. TremD
Table 3 Paracoccidioides isolates used in this study.

\begin{tabular}{|c|c|c|c|}
\hline Isolate & Origin & Country & $\begin{array}{c}\text { Phylogenetic } \\
\text { Species }^{\mathrm{c}}\end{array}$ \\
\hline $\mathrm{PbO1}^{\text {a e } \mathrm{f}}$ & clinical & Brazil - Goiás & Pbo1-like \\
\hline $\mathrm{Ed} 01^{\mathrm{g}}$ & clinical & Brazil - Goiás & Pbo1-like \\
\hline $1578^{9}$ & clinical & Brazil - Goiás & Pbo1-like \\
\hline $\mathrm{Pb} 03^{\mathrm{ad} f \mathrm{hi}}$ & chronic $\mathrm{PCM}^{\mathrm{b}}$ & $\begin{array}{l}\text { Brazil - São } \\
\text { Paulo }\end{array}$ & PS2 \\
\hline $\mathrm{Pb} 4^{d \text { e } f h i}$ & chronic PCM & $\begin{array}{l}\text { Brazil - São } \\
\text { Paulo }\end{array}$ & PS2 \\
\hline $\mathrm{Pb} 2^{\mathrm{d} \text { e } \mathrm{fhi}}$ & chronic PCM & Venezuela & PS2 \\
\hline $\operatorname{Pb}_{i}{ }_{i}^{a d ~ e f ~} h$ & chronic PCM & $\begin{array}{l}\text { Brazil - São } \\
\text { Paulo }\end{array}$ & $\mathrm{S} 1$ \\
\hline$B 339^{d \text { e } f ~ h ~ i ~}$ & chronic PCM & $\begin{array}{l}\text { Brazil - São } \\
\text { Paulo }\end{array}$ & S1 \\
\hline $\mathrm{Pb}^{\mathrm{d} f \mathrm{~h} \mathrm{i}}$ & chronic PCM & Brazil - Paraná & S1 \\
\hline $\mathrm{Pb} 5^{\mathrm{h}} \mathrm{i}$ & chronic PCM & Brazil - Paraná & S1 \\
\hline Utero ${ }^{\mathrm{g}}$ & chronic PCM & Argentina & S1 \\
\hline $\mathrm{Pb} 9^{\mathrm{d} f \mathrm{~h} \mathrm{i}}$ & chronic PCM & $\begin{array}{l}\text { Brazil - São } \\
\text { Paulo }\end{array}$ & S1 \\
\hline $\mathrm{Pb} 11^{\mathrm{d} f \mathrm{~h} \mathrm{i}}$ & acute PCM & Brazil - Paraná & S1 \\
\hline $\mathrm{Pb} 13^{\mathrm{d} f \mathrm{~h} \mathrm{i}}$ & acute PCM & Brazil - Goiás & S1 \\
\hline $\mathrm{Pb} 10^{\mathrm{d} f \mathrm{~h} \mathrm{i}}$ & acute PCM & Peru & S1 \\
\hline $\mathrm{Pb} 14^{\mathrm{df} \mathrm{h} \mathrm{i}}$ & acute PCM & $\begin{array}{l}\text { Brazil - São } \\
\text { Paulo }\end{array}$ & S1 \\
\hline $\mathrm{Pb} 12^{\mathrm{e} \mathrm{h} \mathrm{i}}$ & acute PCM & Argentina & S1 \\
\hline Penguin $^{d f}$ & $\begin{array}{l}\text { penguin } \\
\text { faeces }\end{array}$ & Uruguay & S1 \\
\hline
\end{tabular}

${ }^{\text {a }}$. brasiliensis isolates used in the genome sequencing project - Broad Institute MIT and Harvard.

${ }^{b} P C M$ - paracoccidioidomycosis

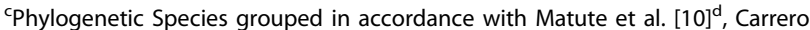
et al. [11] $]^{\mathrm{e}}$, Teixeira et al. [12] ${ }^{f}$ and Figure 7 (this work) ${ }^{\mathrm{g}}$.

hIsolate was also used by Morais et al. [9]

'Revised by Puccia et al. [13].

was detected in all $\mathrm{S} 1$ isolates but not in the Pb01-like ones. TremE was amplified in Pb01-like isolates but not in S1 or PS2 isolates. A $2.3 \mathrm{~kb}$ TremF amplicon was detected in PS2 and S1 isolates. An additional hybridizing band of $\sim 2.0 \mathrm{~kb}$ was detected in $7 \mathrm{~S} 1$ isolates, suggesting the presence of degenerate copies of TremF. In the Pb01-like isolates, a smaller PCR product was observed, but it did not hybridize with the TremF probe. The element TremH was amplified in all isolates we analyzed. Because of low copy-number of TremH in Pb01-like isolates, a longer exposure of the autoradiogram was needed to visualize the hybridizing bands. A strong hybridizing $2.0 \mathrm{~kb}$ band was seen in PS2 and S1 isolates. An additional hybridizing band of $\sim 2.0 \mathrm{~kb}$ was detected in $9 \mathrm{~S} 1$ isolates, suggesting the presence of degenerate copies of TremH.

\section{Transcription of TremA and B elements}

To isolate Trem transcripts, cDNAs from yeast forms were synthesized by RT-PCR using primers from TremA and TremB shown in Additional file 7. The 


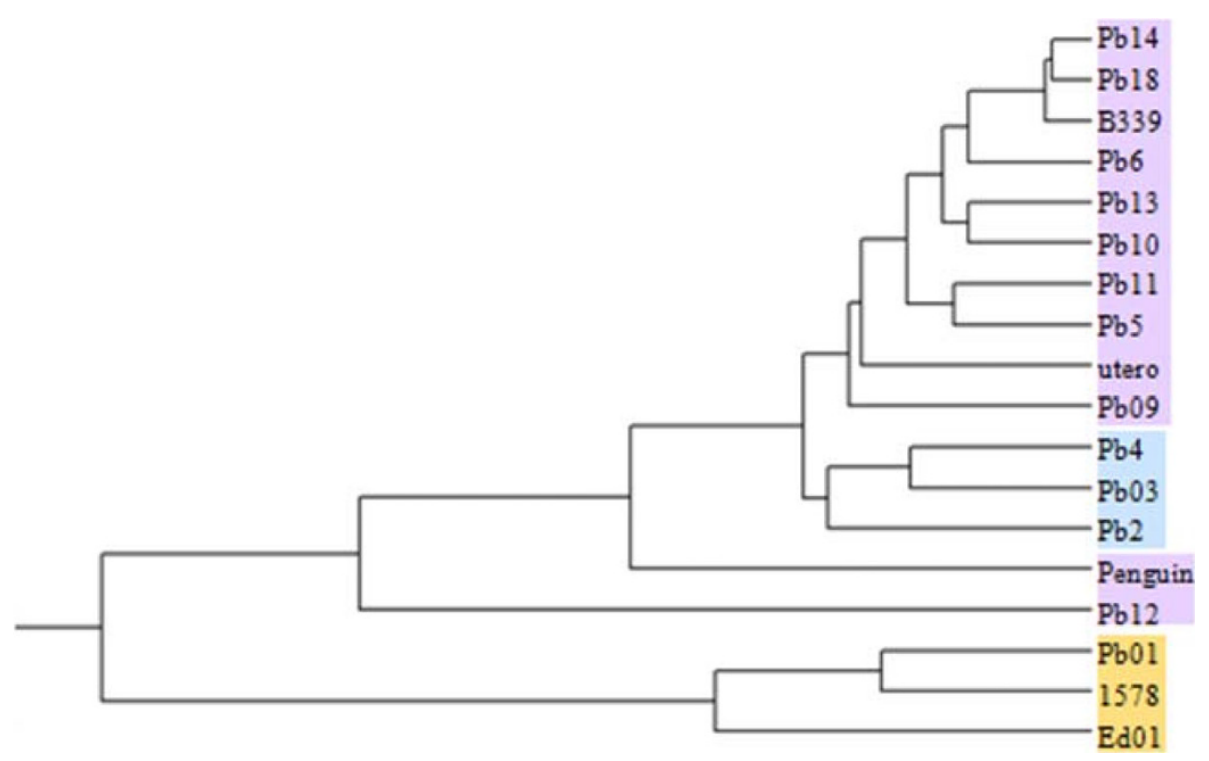

Figure 7 Dendrogram of random amplified polymorphic DNA (RAPD) analysis of 18 . brasiliensis isolates. Dendrogram built from the results of RAPD analysis of genomic DNA of 18 P. brasiliensis isolates using 6 random primers (see Methods). The RAPD analysis allowed clear differentiation of the 18 isolates into 3 distinct groups Pb01-like, S1 and PS2. The Pb01-like isolates are highlighted in salmon, the S1 isolates in lilac and the PS2 isolates in light blue

RT-PCR products were separated by electrophoresis (Additional file 8), purified from the agarose gel and cloned into TA-vectors for sequencing. Transcripts of length corresponding to the unit size of the elements were amplified for Pb03 and Pb18 (P. brasiliensis, phylogenetic species PS2 and S1, respectively), but not for Pb01 (P. lutzii). Recombinant cDNA clones from isolate $\mathrm{Pb} 03$ were sequenced and found to be homologous to TremA (99.6\% identity, NCBI accession number FJ886812) and TremB (99.3\% identity, NCBI accession number - FJ886813), confirming that these elements are transcribed. In conclusion, Trem elements are not silent in the genome.

\section{Discussion}

We have carried out the first systematic search for DNA transposons in the genomic sequence of the dimorphic pathogenic fungus Paracoccidioides, isolates Pb01 ( $P$. lutzii), $\mathrm{Pb} 03$ and $\mathrm{Pb} 18$ (P. brasiliensis, phylogenetic lineages PS2 and S1, respectively) (Assembly 1). The computational search strategy identified approximately 1300 putative transposon sequences (Table 1) in the genome of the 3 isolates, signaling the occurrence of class II TEs in the genus Paracoccidioides. We identified 8 families of transposons of the Tc1/mariner superfamily, based on the analysis of transposases and their associated TIRs and TSDs [20]. With the exception of TremG, in which the TIRs could not be defined, Trem elements are typical Tc1/mariner transposons exhibiting the TIR-transposase-TIR structure. Tc1/mariner superfamily is probably the most widely distributed transposon family in nature, being frequently found in the genomes of filamentous fungi $[17,28]$. However, there have to date been no descriptions of DNA transposons in dimorphic fungi. However, DNA transposons in the genus Aspergillus (subclass Eurotiomycetidae NCBI, "taxonomy browser"), phylogenetically related to the dimorphic fungi, have already been identified $[29,30]$.

Trem elements represent a significant proportion of the genome - around $1 \%$ of the genome of P. brasiliensis (isolates $\mathrm{Pb} 03$ and $\mathrm{Pb} 18$ ) and $0.6 \%$ of P. lutzii (Pb01) indicating their successful proliferation in the genome. It is noteworthy that the majority $(60-70 \%)$ of putative Paracoccidioides DNA transposons correspond to degenerated sequences (probably older insertions) that are difficult to classify; and were not included in this description. Among the remaining sequences, it may be possible to find elements belonging to the MITEs ("Miniature Inverted-repeat Transposable Elements"), a category of small, nonautonomous DNA transposons (100$500 \mathrm{bp}$ ) carrying preserved TIRs found in various organisms, including fungi [31].

Of the 464 Trem copies, 16 (3.5\%) may be potentially autonomous active elements, as judged by the presence of intact TIRs and ORFs coding for transposases. Copies with truncated ORFs but preserved TIRs may be mobilized in trans by the enzyme coded by an intact copy [20]. HTH and DDE domains were preserved in the potentially active Trem elements. As previously observed 


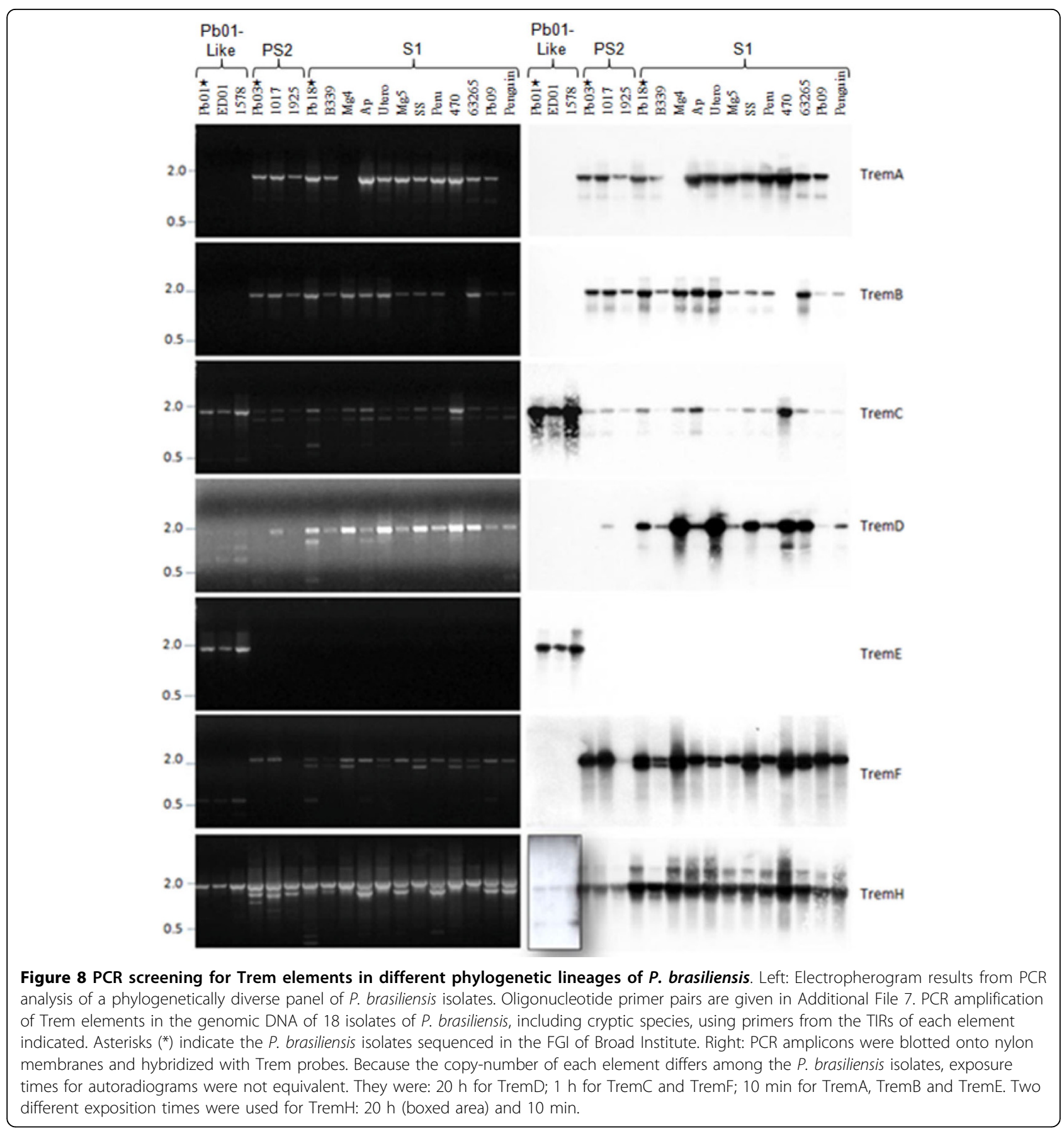

in fungi, the glutamyl residue of the DDE domain was substituted by an aspartyl residue (DDD) $[29,30]$. The currently most known eukaryotic cut-and-paste transposons are DDE-superfamilies, PiggyBac and Tc1/Mariner being the only 2 superfamilies encoding DDD-transposases. Conservation of the triad signature (DDD) in Tc1/ mariner transposons may reflect a catalytic mechanism similar to that described for the DDE motif, which probably coordinate divalent metal ions to promote catalysis of DNA cleavage and ligation.
The CENPB domain was found in TremA, TremB and TremF elements. This domain has been identified in mammalian centromeric proteins and eukaryotic transposases, but not in any archaeal, bacterial or plant proteins. It may be implicated in the recognition of the terminal inverted repeats of transposons. The functional similarity between CENPB domains could be the result of domestication of transposons by the host genome to act as its own genes or regulatory elements [29,32-34]. The N-terminal HTH domain, also found in the 
truncated elements TremC and TremE, has similarity with the "pipsqueak" DNA-binding domain of Drosophila, a family of eukaryotic proteins that recognize and bind to DNA sequences [35]. The terminal inverted repeats were identified in 7 of the 8 Trem elements. The inverted regions had sizes compatible with those of other elements belonging to the Tc1/mariner superfamily described in fungi, and also had a $17 \mathrm{bp}$ internal direct repeat, as previously reported $[17,29,36]$. It was suggested that this direct repetition could be the recognition and binding of the HTH and CENPB transposase domains [37].

An interesting finding was the presence of conserved $\mathrm{Cx} 2 \mathrm{Cx} 4 \mathrm{Hx} 4 \mathrm{C}(\mathrm{CCHC})$ motifs indicating zinc finger (ZF) domains in the $\mathrm{C}$-terminal ends of transposases of at least 3 Trem elements (TremA, TremB and TremD). The ZF, which is present in a wide range of proteins, forms a structure stabilized by co-ordination of a divalent zinc cation [Zn (II)], with cysteine or histidine residues as ligands. ZF are structurally diverse and present among proteins with a broad range of cellular functions, such as replication and repair, transcription and translation, metabolism and signaling, cell proliferation and apoptosis. Krishna et al. [38], who described 8 fold groups of ZF, discussed their similarities and differences with functional implications. The fold group 1 consists of 2 families: $\mathrm{C} 2 \mathrm{H} 2$ fingers and IAP (inhibitor of apoptosis) domains. IAP contains a CCHC pattern that coordinates a zinc ion, a pattern similar to that found at the $\mathrm{C}$-terminal region of Trem elements transposases. The ZF is responsible for binding to DNA or RNA in some proteins, or for the protein-protein interaction in others. Ohta et al. [39] described transposases encoded by transposable elements of the Insertion Sequences 1 (IS1 family) of Escherichia coli as having N-terminal regions containing a $\mathrm{C} 2 \mathrm{C} 2 \mathrm{ZF}$ motif. They showed that the ZF motif, as well as the HTH motif, is essential for transposition promoted by IS1transposase because of their involvement in binding specifically to TIRs. IS1 transposases with an amino acid substitution in the HTH or ZF motif lose the ability to promote transposition, indicating that 2 domains are responsible for TIR-specific DNA binding in promoting transposition. It is likely that the 2-domain structure is required for binding to TIRs, not only in IS1transposase, but also in transposases encoded by some other transposable elements, in order to place a catalytic domain of transposases in a region near the TIR end, where strand transfer reactions occur in transpososomes. These observations suggest the possibility that transposases encoded by some elements have 2 domains involved in the TIR-specific DNA-binding. In eukaryotes, a Drosophila transposable element $\mathrm{P}$ encodes a transposition inhibitor that has a $\mathrm{CCHC}$ region forming a $\mathrm{ZF}$ that plays an important role in the binding to the TIR sequences at the termini of the P element [38,39].

When transposons move along the genome, footprints known as TSDs ("Target Site Duplications") can be formed that flank the element. Most transposon superfamilies may have a characteristic TSD with a specific size and sequence [20]. TSDs were found in 6 out of the 8 Trem elements. It was not possible to determine the TIRs in TremG and consequently find the flanking TSD. The copies of TremH are truncated and probably inactivated, resulting in loss or alteration of the TSD, consequently preventing its identification.

As the DNA transposons of the Tc1/mariner superfamily have the dinucleotide TA as their insertion site, they can be inserted within virtually any genomic region. However, regional preferences have been observed for many elements which are inserted in specific regions of the genome [40]. The comparison of the number and identity of nested insertions within individual transposons has been used to estimate the relative ages of transposons. It is reasonable to assume that the older elements are subjected to interruptions by more recently active elements. Older elements should therefore have higher proportions of nested insertions than younger elements. Assuming that DNA transposon insertions occur randomly throughout the genome, there should be a higher proportion of nested insertions for older Trem elements than for younger ones. The following evidence could support the hypothesis of recent transposition and expansion of Trem elements in the genome: (a) the absence of nested Trem copies inside other transposons; (b) the presence of intact open reading frames; and (c) the presence of highly similar copies in some Trem elements. Analysis of Southern blots and chromoblots of genomic DNA from isolates from 2 different species, P. lutzii ( $\mathrm{Pb} 01)$ and P. brasiliensis ( $\mathrm{Pb} 03$ and $\mathrm{Pb} 18)$, corroborates the in silico data in relation to the presence and distribution of Trem elements in these genomes.

Trem elements do not appear to have been acquired simultaneously by the Paracoccidioides sequenced genomes, as indicated by finding of different copy-numbers among Trem families. The elements TremC and TremH are present in a large number of copies, most of which are truncated elements with stop codons. Unlike TremC and TremH, TremD is only present in 5 copies, 4 of which have integral ORFs, just in isolate Pb18 (P. brasiliensis, phylogenetic species S1). This pattern is compatible with a recent insertion in the genome, as TremD would not yet have been inactivated by the genome defense system.

Several recent studies have proposed the existence of different phylogenetic species in the taxon P. brasiliensis. Matute et al. [10] analyzed the genetic structure of 
$65 P$. brasiliensis isolates, concluding that they can be grouped into 3 distinct phylogenetic species: S1 (including isolate $\mathrm{Pb} 18$ ), PS2 (including isolate Pb03) and PS3 (composed exclusively of Colombian isolates). In a study of 21 P. brasiliensis isolates, 14 of which had been included in the above study, Carrero et al. [11] reached a similar conclusion for all the isolates except isolate $\mathrm{Pb} 01$, which they suggested was a new phylogenetic species in the genus Paracoccidioides. Recently, Teixeira et al. [12], analyzing 88 isolates of the fungus, found that 17 were genotypically similar, belonging to the Pb01-like group. They estimated that the S1/PS2/PS3 species clade and the Pb01-like new species, for which the name $P$. lutzii was proposed, shared a common ancestor approximately 32 million years ago.

We used PCR to analyze the distribution of Trem elements in 17 clinical isolates and one environmental isolate formerly known as P. brasiliensis. Previous reports using RAPD for typing $P$. brasiliensis indicated that the use of certain primers and conditions allowed the discrimination of isolates with very low genetic similarity clustering the most similar ones. The primers chosen discriminated of $P$. brasiliensis isolates into groups with very low genetic similarity indexes $(17-35 \%$ when the $\mathrm{Pb} 01$ isolate was included or at least $50-60 \%$ when it was not) $[5,26,41]$. Probably for this reason our RAPD analysis resulted in the clustering of isolates previously known to belong to a certain phylogenetic species (Pb01-like, S1 and/or PS2), which could be a reference for the characterization of those of unknown genetic relatedness (Table 3, see isolates Ed01, 1578 and utero). The 18 isolates consisted of the sequenced $\mathrm{Pb} 01$ and 2 Pb01-like isolates (P. lutzii) [12]; 3 isolates previously classified as PS2 (including isolate $\mathrm{Pb03}$ ); and 12 isolates identified as S1 (including Pb18). PS3, the fourth known phylogenetic species reported to be exclusively in Colombia was not represented [10]. The isolates were grouped in accordance with Matute et al.[10], Carrero et al. [11], Teixeira et al. [12] and the results of RAPD analysis (Figure 7) [27]. Isolates Penguin and $\mathrm{Pb} 12$ are known to be $P$. brasiliensis (S1) but do not cluster with the other S1 isolates (Figure 7). These isolates come from different geographic regions, Uruguay and Argentina, while the majority is Brazilian (13 isolates, Table 3 ). It is interesting that a fungal associated feature that has been reported to correlate by RAPD branching or clustering of Paracoccidioides refers to the isolate's geographic origin [13]. The non-grouping of all S1 isolates would be explained by the fact that multilocus sequencing studies employ nuclear genes while RAPD would arbitrarily reveal polymorphic regions along the genomes, including intergenic and/or repetitive sequence.

The DNA transposons TremC and TremH were identified in all the isolates tested, indicating that these elements would have already been present in a hypothetical common ancestor rather than have been acquired horizontally after the 3 phylogenetic species diverged from one another. In contrast, TremE was only found in Pb01 and Pb01-like isolates, and TremA, TremB, and TremF were found in the S1 and PS2 isolates, while the element TremD was almost exclusively found in S1 isolates, suggesting that these DNA transposons could have been acquired horizontally after separation of the 3 phylogenetic species. Although TremD and TremE share approx $70 \%$ similarity at the amino acid level, they were found exclusively in the $\mathrm{S} 1$ and Pb01-like isolates, respectively, which are phylogenetically distant. This suggests that these elements would have been acquired by horizontal transfer after the split between S1/PS2 ( $P$. brasiliensis) and Pb01-like species (P. lutzii). This hypothesis is supported by the finding of hypothetical protein sequences of $A$. capsulatum in GenBank (accession numbers XM 001537507 and XM 001543155) that yielded an e-value of 0.0 for the elements TremD and TremE, respectively (i.e. their sequences are identical or have at least $80 \%$ identity). However, the absence of TremD and TremE could also be explained by the loss of these elements in some strains.

Transcription of TremA and TremB suggests that they could be active elements. When transposing, the elements generate double-strand DNA breaks, which are repaired by the cellular machinery [42]. Hence, in addition to the direct effects of transposition, such as gene inactivation, recombination events promoted by repair of the double strand would also take place. Transposition of these elements could be a factor involved in the genetic variability observed in the $P$. brasiliensis species complex. The identification of active elements would serve as a basis for the development of mutational tools, as transposons could be efficient vectors for modifications.

\section{Conclusions}

The strategy developed in the present work proved effective in identifying and characterizing DNA transposons in the newly generated sequence assemblies of isolates of 3 phylogenetic species of the P. brasiliensis complex. The most intact insertions allowed the classification of 7 new families and a new subfamily of elements (TremA - H) that share identity with the Tc1/ mariner superfamily, distinct lineages not yet identified in dimorphic fungi. Three full-length, potentially functional autonomous elements were characterized (Trem A, B and D). Further studies to show their active transposition would be interesting. The phylogenetic analysis supports the hypothesis that Trem families derived from a single ancestral sequence and split into 2 lineages (TremA - F; TremG - H). The occurrence of Trem 
families is unequal in the genomic sequence of the isolates $\mathrm{Pb} 01, \mathrm{~Pb} 03$ and $\mathrm{Pb} 18$ and experimental evidence from the other 15 typed isolates indicated that some Trem families (TremC and $\mathrm{H}$ ) were shared while other Trem families were harbored exclusively by Pb01-like species (P. lutzii) (TremE) or S1/PS2 species (P. brasiliensis) (TremA, B, D, and F). It would be interesting to verify the occurrence of distinct Trem families in a larger number of $P$. lutzii and P. brasiliensis isolates from different origins (either clinical or environmental), and from different geographic regions, also including the phylogenetic species PS3, not represented here, in such a way as to expand and explore the potential of these newly presented genetic agents as sources of phylogenetic and epidemiological knowledge.

\section{Methods}

\section{Bioinformatic analysis}

A retrieval based on specific keywords (transposase, transposons, transposable) was performed against the $P$. brasiliensis Functional and Differential Genome Project EST annotated database (PbDBEST) http://www.biomol. unb.br/[15,24] to identify sequences containing particular annotation terms related to transposable elements. At the time of writing, 2 contigs have been identified: Contigs 530 (673 bp) and 1938 (637 bp), GenBank accession numbers CN240498 and CN247880, respectively.

These contigs were further used in similarity searches using BLAST algorithm [38] (specifically blastn and tblastx with default parameters) against the first draft of the genomic sequence of $P$. brasiliensis isolates $\mathrm{Pb} 03$, $\mathrm{Pb} 01$ and Pb18 released by the Fungal Genome Initiative Project (FGI) of The Broad Institute http://www.broad. mit.edu.

\section{Databases}

The $P$. brasiliensis genomic sequences of isolates $\mathrm{Pb} 01$, $\mathrm{Pb} 03$ and Pb18 from the Fungal Genome Initiative Project (FGI) of The Broad Institute were downloaded (Paracoccidioides brasiliensis Database) http://www. broad.mit.edu/annotation/genome/paracoccidioides_brasiliensis/Downloads.html and used in the in silico analysis described in this work. The locally compiled database of $P$. brasiliensis sequences, built by parsing sequences from the original database, was used for sequence similarity searches, using the BLAST [43] algorithm.

\section{Transposon mapping and TIR identification}

To identify and map transposable elements in the genomic sequences of $P$. brasiliensis isolates $\mathrm{Pb} 03, \mathrm{~Pb} 01$ and $\mathrm{Pb} 18$, an approach was developed that we called "ClassSpecific Method for transposable element identification".
This method takes into account the advantage of the knowledge of particular genomic features characteristic of specific classes of the elements to aid their identification.

The homology-based method we developed identifies transposable elements by comparing genomic query sequences (Pb01, Pb03 and Pb18) against themselves. Sequence comparison by BLAST algorithm took into account query hits identified in a search using only the top strand (-S 1 parameter) to search against database, a word size of 7 . To prevent long high-scoring segment pairs (HSPs) being broken up into smaller ones because of low-complexity segments and repeats, the default filter (DUST) was turned off. Specific databases containing each genomic sequence of $P$. brasiliensis isolates were built converting the DNA sequence into its reversecomplement counterpart. Similarity results that emerge from this self comparison were parsed by 2 in-house developed PERL (Practical Extraction and Report Language) scripts. These scripts specifically convert the standard BLAST output into a tabular format and allow the identification of pairs of inverted repeats at approximately $2.5 \mathrm{~Kb}$ from each other (i.e. the average distance between TIRs of Tc1/mariner elements).

The sequence stretches containing flanking inverted repeats identified using this initial criteria were finally compared in terms of the putative gene products encoded against NCBI nr protein database to identify putative transposable elements.

\section{Annotation}

The annotation and graphical output of the supercontigs were performed using ARTEMIS [44] software http:// www.sanger.ac.uk/Software/Artemis/ and 4 in-house developed PERL scripts to analyze and format the results.

Classification of the DNA transposons follows the nomenclature previously established, and families and subfamilies were disclosed according to the standard principles of TE classification [20,45-47]. In this report we define the DNA transposon family by DNA sequence as described by Wicker at al. [20]: 2 elements belong to the same family if they share $80 \%$ (or more) sequence identity in at least $80 \%$ of their coding region or internal domain or within their terminal repeats (or both). All consensus sequences (Additional file 9) were submitted to Repbase using the Repeat Masking algorithm to identify related elements http://www.girinst.org/censor/index.php.

\section{Multiple sequence alignment and phylogenetic inference} Global multiple sequence alignments using amino acid sequences from identified transposases and nucleotide sequences from identified transposons were performed using the ClustalX algorithm [48], followed by visual 
inspection and manual adjustment with SeaView [49] http://pbil.univ-lyon1.fr/software/seaview.html and GeneDoc http://www.psc.edu/biomed/genedoc/.

Phylogenetic reconstruction of the sequences aligned by ClustalW [48] were performed using Neighbor-joining method implemented in MEGA 4 [50]. Trees were constructed using the equal-input model with 5000 bootstrap replicates. Branch lengths reflect the sequence divergence (see the scale bar). The amino acid sequences used in the phylogenetic reconstruction are available at: http://www. girinst.org/repbase/update/browse.php with the follow locus names: Mariner-6_AN, Mariner-3_AF, Mariner7_AN, Mariner-1_AF, Mariner2_AO, Mariner3_AO, Mariner-5_AF, Mariner-7_AF, Mariner-3a_AN, Mariner3_AN, Mariner-1_AN, Mariner-4_AN, Mariner-5_AN, Mariner5_AO. The amino acid sequences from pogo_ DM and Pot2_MG (NCBI accession number S20478 and XP364943, respectively) were also used.

\section{$P$. brasiliensis isolates and growth conditions}

The characteristic P. brasiliensis isolates are listed in Table 3. Fungal isolates were maintained by periodic subculturing in PYG medium (yeast extract 5 g, bactopeptone $10 \mathrm{~g}$, dextrose $15 \mathrm{~g}$, and Agar $15 \mathrm{gl}^{-1}, \mathrm{pH}$ 6.3) at $35-37^{\circ} \mathrm{C}[8]$.

\section{DNA and RNA extraction}

Total DNA was extracted from the yeast culture following previously described protocols involving maceration of frozen cells, with minor modifications [51]. Total RNA was extracted from the yeast cultures as previously reported [52].

\section{PCR}

PCR reactions were carried out on $10 \mathrm{ng}$ of DNA in a $50 \mu \mathrm{L}$ reaction mixture containing $10 \mathrm{mM}$ Tris- $\mathrm{HCl}, \mathrm{pH}$ 9, $1.5 \mathrm{mM} \mathrm{MgCl} 2,50 \mathrm{mM} \mathrm{KCl}, 100 \mathrm{mM}$ of each dNTP, $100 \mathrm{pmol}$ of each oligonucleotide and 1 unit of Taq DNA Polymerase (Promega). Each PCR was carried out in a programmable temperature controller (Eppendorf) for 25 cycles. The cycling conditions were as follows: denaturing at $94^{\circ} \mathrm{C}$ for $1 \mathrm{~min}$, annealing at the $\mathrm{Tm}$ for each primer for $30 \mathrm{~s}$, and extension at $72^{\circ} \mathrm{C}$ for $1 \mathrm{~min}$. At the end of the $25^{\text {th }}$ cycle, the heat-denaturing step was omitted and extension was allowed to proceed at $72^{\circ} \mathrm{C}$ for $5 \mathrm{~min}$. The primers and respective Trem are listed in Additional file 7.

\section{RAPD analysis}

RAPD analysis was performed as previously described $[5,26,41]$, using the primers OPG03 (GAGCCCTCCA), OPG11 (TGCCCGTCGT), OPG15 (ACTGGGACTC), OPG16 (AGCGTCCTCC), OPG18 (GGCTCATGTG) and OPO06 (CCACGGGAAG).
PCR reactions were carried out in $10 \mathrm{mM}$ Tris- $\mathrm{HCl}$, $\mathrm{pH} 9,2 \mathrm{mM} \mathrm{MgCl} 2,50 \mathrm{mM} \mathrm{KCl}, 200 \mathrm{mM}$ of each $\mathrm{dNTP}$, and $0.25 \mathrm{mM}$ of each oligonucleotide in a $20 \mu \mathrm{L}$ reaction mixture containing 1 unit of Taq DNA Polymerase (Phoneutria). The cycling conditions were as follows: denaturing at $94^{\circ} \mathrm{C}$ for 3 min followed by 40 cycles of denaturing at $94^{\circ} \mathrm{C}$ for $45 \mathrm{~s}$, annealing at $36^{\circ} \mathrm{C}$ for 45 $\mathrm{s}$ and extension at $72^{\circ} \mathrm{C}$ for $105 \mathrm{~s}$. At the end of the $40^{\text {th }}$ cycle, the heat-denaturing step was omitted and extension was allowed to proceed at $72^{\circ} \mathrm{C}$ for $3 \mathrm{~min}$. The products were separated by polyacrylamide gel electrophoresis and silver stained (5\% silver stain). Digitized gel images were analyzed by LabImage version 2.7.1, and dendrograms were generated by TFPGA software version 1.3.

\section{Transcriptional analysis}

First- and second-strand cDNAs were prepared using SuperScript One-Step reverse transcriptase PCR (RTPCR) with Platinum Taq according to the manufacturer's instructions (Life Technologies). Specific forward or reverse primers based on the nucleotide sequences of the elements TremA and TremB were used with oligo (dT) to amplify sequences by PCR. Genomic DNA false positive results were excluded by performing the reaction in the absence of reverse transcriptase, and with a treatment of RNA samples, prior to the RT-PCR reaction, with DNase I, RNase free.

\section{Cloning and sequencing}

TremA and TremB genomic and cDNA amplicons were then cloned into plasmid pGEM-T Easy vector (Promega) and transformed into Escherichia coli DH5 $\alpha$ competent cells. Nucleotide sequences of cDNA clones were determined by the dideoxynucleotide chain termination method, using BigDye Terminator cycle sequencing chemistry (Applied Biosystems) in an ABI PRISM 377 DNA sequencer.

\section{Blotting analysis}

For Southern blot analysis, PCR amplifications or DNA samples digested with the restriction enzyme BglII, were separated by electrophoresis on $0.8 \%$ agarose gels and stained with ethidium bromide $(0.5 \mu \mathrm{g} / \mathrm{mL})$. For chromoblot analysis, the separation of chromosomesized P. brasiliensis DNA molecules by PFGE (pulsed field gel electrophoresis) was performed as described by Feitosa et al. [8]. The agarose gels were incubated with $0.25 \mathrm{M} \mathrm{HCl}$ for $30 \mathrm{~min}$, denatured with $0.5 \mathrm{M}$ $\mathrm{NaOH} / 1 \mathrm{M} \mathrm{NaCl}$ for $30 \mathrm{~min}$, neutralized with $1 \mathrm{M}$ Tris-base $/ 0.5 \mathrm{M} \mathrm{NaCl}$ for $20 \mathrm{~min}$ and transferred onto nylon membranes in $20 \times \mathrm{SSC}(0.15 \mathrm{M} \mathrm{NaCl}$ and $0.015 \mathrm{M}$ sodium citrate). The membranes were prehybridized in a solution containing $50 \%$ formamide- $5 \times, 5$ 
$\times$ SSC, $0.5 \%$ Denhardt's solution, $0.1 \mathrm{mg} / \mathrm{mL}$ salmon sperm DNA and $0.1 \mathrm{mg} / \mathrm{mL}$ tRNA at $42^{\circ} \mathrm{C}$ for $1 \mathrm{~h}$, and then hybridized overnight at the same temperature with a ${ }^{32} \mathrm{P}$-labeled probe. The probes used in the hybridization experiments were derived from the internal region of each element, including the ORF. Following hybridization, the membranes were washed 3 times (30 min each) in $2 \times$ SSC containing $0.1 \%$ SDS at $42^{\circ} \mathrm{C}, 1 \times$ SSC containing $0.1 \%$ SDS and $0.1 \times$ SSC containing $0.1 \% \mathrm{SDS}$ at $56^{\circ} \mathrm{C}$, before being exposed to X-ray film.

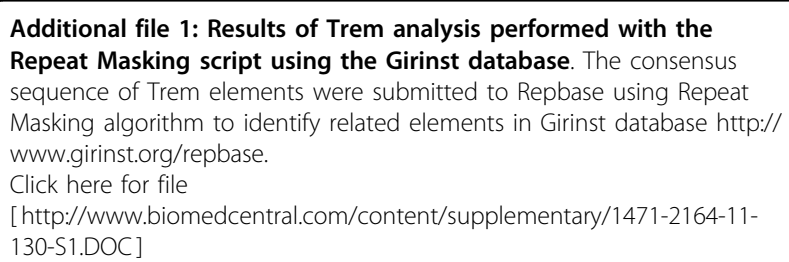

Additional file 3: Distribution of Trem elements in the genome Information of each supercontig: GenBank accession number, size and number of Trem elements. In the column ORF are presented only the insertions of Trem elements that show complete, non truncated ORFs. Click here for file

[http://www.biomedcentral.com/content/supplementary/1471-2164-11130-S3.XLS]

Additional file 4: Supercontig view showing the distribution of Trem elements in the genome of $P$. brasiliensis isolate $\mathrm{Pb03}$. Each supercontig is represented by a dashed line in scale. Below each dashed line the Trem insertions along the supercontig are represented. The GenBank accession number of contigs is indicated in parentheses. The coordinates of each Trem element (genome location, length, TIR) displayed in the supercontig view are accessible in Additional file 1. Click here for file

[http://www.biomedcentral.com/content/supplementary/1471-2164-11130-S4.PNG ]

Additional file 5: Supercontig view showing the distribution of Trem elements in the genome of $P$. brasiliensis isolate $\mathrm{Pb} 18$. Each supercontig is represented by a dashed line in scale. Below each dashed line the Trem insertions along the supercontig are represented. The GenBank accession number of contigs is indicated in parentheses. The coordinates of each Trem element (genome location, length, TIR) displayed in the supercontig view are accessible in Additional file 1. Click here for file

[http://www.biomedcentral.com/content/supplementary/1471-2164-11130-S5.PNG ]

Additional file 6: Supercontig view showing the distribution of Trem elements in the genome of $P$. brasiliensis isolate Pb01. Each supercontig is represented by a dashed line in scale. Below each dashed line the Trem insertions along the supercontig are represented. The GenBank accession number of contigs is indicated in parentheses. The coordinates of each Trem element (genome location, length, TIR) displayed in the supercontig view are accessible in Additional file 2 Click here for file

[http://www.biomedcentral.com/content/supplementary/1471-2164-11130-S6.PNG ]
Additional file 7: Sequences of primers used to amplify Trem elements by PCR and RT-PCR. Table with the sequence of the primers used to amplify Trem elements by PCR and RT-PCR.

Click here for file

[http://www.biomedcentral.com/content/supplementary/1471-2164-11130-S7.DOC ]

Additional file 8: Transcription of TremA and TremB elements in $P$. brasiliensis isolates. RT-PCR was carried out on total RNA from the yeast form of isolates $\mathrm{Pb} 01, \mathrm{Pb03}$ and $\mathrm{Pb} 18$ using specific primers for the ORFs of TremA and TremB (see Additional file 7).

Click here for file

[http://www.biomedcentral.com/content/supplementary/1471-2164-11130-S8.PPT]

Additional file 9: Consensus sequences of Trem element. The nucleotide consensus sequence of Trem elements identified. Click here for file

[http://www.biomedcentral.com/content/supplementary/1471-2164-11130-S9.TXT]

\section{Abbreviations}

RAPD: random amplification of polymorphic DNA; RFLP: restriction fragment length polymorphism; GCPRS: genealogic concordance phylogenetic species recognition; TE: transposable element; TIR: terminal inverted repeat; HTH: Helix-Turn-Helix_pipsqueak; TSD: target site duplications; MITE: miniature inverted-repeat transposable element; FGl: Fungal genome initiative; GIRI: genetic information research institute; Trem: transposable element mariner; ZF: zinc finger; HSPs: high-scoring segment pairs; DUST: default filter; PERL: practical extraction and report language

\section{Acknowledgements}

We would like to thank Dr. Mia Champion, the research publication coordinator of the Fungal Genome Initiative Projects of the Broad Institute MIT and Harvard for carefully reading and presenting relevant suggestions for both the work and the manuscript. This work was supported by grants from FAPEMIG and CNPq (Brazil) to PSC and from FAPESP and CNPq (Brazil) to JFS. MMM and RRMB were awarded doctoral fellowships by CAPES and FAPESP, respectively; ACPG and PCS were awarded master's fellowships by CNPq and CAPES, respectively; and TZ is an undergraduate student awarded a Scientific Initiation scholarship by FAPESP. This work used computational resources made available through an NIH Grant (TW007012) and FAPEMIG (REDE-186/08) to JCR.

\section{Author details}

'Departamento de Microbiologia, Instituto de Ciências Biológicas, Universidade Federal de Minas Gerais, 31270-901 Belo Horizonte, MG, Brazil. ${ }^{2}$ Departamento de Microbiologia, Imunologia e Parasitologia, Escola Paulista de Medicina, Universidade Federal de São Paulo, 04023-062 São Paulo, SP, Brazil. ${ }^{3}$ Laboratório de Biologia Molecular, Instituto de Ciências Biológicas, Universidade Federal de Goiás, 74001-970 Goiânia, GO, Brazil. " Laboratório de Biologia Molecular, Instituto de Ciências Biológicas, Universidade de Brasília, 70910-900 Brasília, DF, Brazil. ${ }^{5}$ Centro de Pesquisas René Rachou, FIOCRUZ, 30190-002 Belo Horizonte, MG, Brazil.

\section{Authors' contributions}

MMM participated in the experimental design, bioinformatics analysis and annotation, experiments on physical mapping and transcriptional analysis, and manuscript preparation. TZ, PCS, RRMB and ACPG participated in and supported experiments in physical mapping, cloning and sequencing, transcriptional and RAPD analysis. MSSF, MB, CMSA and RP were the principal investigators for the transcriptome and genome projects of $P$. brasiliensis and also participated in manuscript preparation. JCR designed and performed the bioinformatics analysis. JFS and PSC designed, coordinated, supervised and participated in the interpretation, discussion of results and manuscript preparation. All authors read and approved the final manuscript.

Received: 3 September 2009

Accepted: 23 February 2010 Published: 23 February 2010 


\section{References}

1. Brummer E, Castaneda E, Restrepo A: Paracoccidioidomycosis: an update. Clin Microbiol Rev 1993, 6(2):89-117.

2. Franco M, Montenegro MR, Mendes RP, Marques SA, Dillon NL, Mota NG: Paracoccidioidomycosis: a recently proposed classification of its clinical forms. Rev Soc Bras Med Trop 1987, 20(2):129-132.

3. Coutinho ZF, Silva D, Lazera M, Petri V, Oliveira RM, Sabroza PC, Wanke B: Paracoccidioidomycosis mortality in Brazil (1980-1995). Cad Saude Publica 2002, 18(5):1441-1454.

4. Leclerc MC, Philippe H, Gueho E: Phylogeny of dermatophytes and dimorphic fungi based on large subunit ribosomal RNA sequence comparisons. J Med Vet Mycol 1994, 32(5):331-341.

5. Soares CM, Madlun EE, da Silva SP, Pereira M, Felipe MS: Characterization of Paracoccidioides brasiliensis isolates by random amplified polymorphic DNA analysis. J Clin Microbiol 1995, 33(2):505-507.

6. Nino-Vega GA, Calcagno AM, San-Blas G, San-Blas F, Gooday GW, Gow NA RFLP analysis reveals marked geographical isolation between strains of Paracoccidioides brasiliensis. Med Mycol 2000, 38(6):437-441.

7. Cano MI, Cisalpino PS, Galindo I, Ramirez JL, Mortara RA, da Silveira JF: Electrophoretic karyotypes and genome sizing of the pathogenic fungus Paracoccidioides brasiliensis. J Clin Microbiol 1998, 36(3):742-747.

8. Feitosa Ldos S, Cisalpino PS, dos Santos MR, Mortara RA, Barros TF, Morais FV, Puccia R, da Silveira JF, de Camargo ZP: Chromosomal polymorphism, syntenic relationships, and ploidy in the pathogenic fungus Paracoccidioides brasiliensis. Fungal Genet Biol 2003, 39(1):60-69.

9. Morais FV, Barros TF, Fukada MK, Cisalpino PS, Puccia R: Polymorphism in the gene coding for the immunodominant antigen gp43 from the pathogenic fungus Paracoccidioides brasiliensis. J Clin Microbio/ 2000, 38(11):3960-3966.

10. Matute DR, McEwen JG, Puccia R, Montes BA, San-Blas G, Bagagli E, Rauscher JT, Restrepo A, Morais F, Nino-Vega G, et al: Cryptic speciation and recombination in the fungus Paracoccidioides brasiliensis as revealed by gene genealogies. Mol Biol Evol 2006, 23(1):65-73.

11. Carrero LL, Nino-Vega G, Teixeira MM, Carvalho MJ, Soares CM, Pereira M, Jesuino RS, McEwen JG, Mendoza L, Taylor JW, et al: New Paracoccidioides brasiliensis isolate reveals unexpected genomic variability in this human pathogen. Fungal Genet Biol 2008, 45(5):605-612.

12. Teixeira MM, Theodoro RC, de Carvalho MJ, Fernandes L, Paes HC, Hahn RC, Mendoza L, Bagagli E, San-Blas G, Felipe MS: Phylogenetic analysis reveals a high level of speciation in the Paracoccidioides genus. Mol Phylogenet Evol 2009, 52(2):273-83.

13. Puccia R, McEwen JG, Cisalpino PS: Diversity in Paracoccidioides brasiliensis. The PbGP43 gene as a genetic marker. Mycopathologia 2008, 165(4-5):275-287

14. Goldman GH, dos Reis Marques E, Duarte Ribeiro DC, de Souza Bernardes LA, Quiapin AC, Vitorelli PM, Savoldi M, Semighini CP, de Oliveira RC, Nunes LR, et al: Expressed sequence tag analysis of the human pathogen Paracoccidioides brasiliensis yeast phase: identification of putative homologues of Candida albicans virulence and pathogenicity genes. Eukaryot Cell 2003, 2(1):34-48.

15. Felipe MS, Andrade RV, Petrofeza SS, Maranhao AQ, Torres FA Albuquerque $\mathrm{P}$, Arraes FB, Arruda M, Azevedo MO, Baptista AJ, et al: Transcriptome characterization of the dimorphic and pathogenic fungus Paracoccidioides brasiliensis by EST analysis. Yeast 2003, 20(3):263-271.

16. Felipe MS, Torres FA, Maranhao AQ, Silva-Pereira I, Pocas-Fonseca MJ, Campos EG, Moraes LM, Arraes FB, Carvalho MJ, Andrade RV, et al: Functional genome of the human pathogenic fungus Paracoccidioides brasiliensis. FEMS Immunol Med Microbiol 2005, 45(3):369-381.

17. Daboussi MJ, Capy P: Transposable elements in filamentous fungi. Annu Rev Microbiol 2003, 57:275-299.

18. Hua-Van A, Le Rouzic A, Maisonhaute C, Capy P: Abundance, distribution and dynamics of retrotransposable elements and transposons: similarities and differences. Cytogenet Genome Res 2005, 110(1-4):426-440.

19. Daboussi MJ: Fungal transposable elements and genome evolution. Genetica 1997, 100(1-3):253-260.

20. Wicker T, Sabot F, Hua-Van A, Bennetzen JL, Capy P, Chalhoub B, Flavell A, Leroy $\mathrm{P}$, Morgante $\mathrm{M}$, Panaud $\mathrm{O}$, et al: A unified classification system for eukaryotic transposable elements. Nat Rev Genet 2007, 8(12):973-982.

21. Kapitonov W, Jurka J: A universal classification of eukaryotic transposable elements implemented in Repbase. Nat Rev Genet 2008, 9(5):411-412, author reply 414
22. Goodwin TJ, Butler MI, Poulter RT: Cryptons: a group of tyrosinerecombinase-encoding DNA transposons from pathogenic fungi. Microbiology 2003, 149(Pt 11):3099-3109.

23. Nascimento E, Martinez R, Lopes AR, de Souza Bernardes LA, Barco CP Goldman MH, Taylor JW, McEwen JG, Nobrega MP, Nobrega FG, et al: Detection and selection of microsatellites in the genome of Paracoccidioides brasiliensis as molecular markers for clinical and epidemiological studies. J Clin Microbiol 2004, 42(11):5007-5014.

24. Felipe MS, Andrade RV, Arraes FB, Nicola AM, Maranhao AQ, Torres FA, Silva-Pereira I, Pocas-Fonseca MJ, Campos EG, Moraes LM, et al: Transcriptional profiles of the human pathogenic fungus Paracoccidioides brasiliensis in mycelium and yeast cells. J Biol Chem 2005, 280(26):24706-24714

25. Jurka J, Kapitonov W, Pavlicek A, Klonowski P, Kohany O, Walichiewicz J: Repbase Update, a database of eukaryotic repetitive elements. Cytogenet Genome Res 2005, 110(1-4):462-467.

26. Motta TR, Moreira-Filho CA, Mendes RP, Souza LR, Sugizak MF, Baueb S, Calich VL, Vaz CA: Evaluation of DNA polymorphisms amplified by arbitrary primers (RAPD) as genetically associated elements to differentiate virulent and non-virulent Paracoccidioides brasiliensis isolates. FEMS Immunol Med Microbiol 2002, 33(3):151-157.

27. Guerra ACP: Caracterização molecular de isolados clínicos e ambientais do fungo patogênico Paracoccidioides brasiliensis por RAPD e PFGE. Belo Horizonte: Universidade Federal de Minas Gerais 2006

28. Plasterk RH, Izsvak Z, Ivics Z: Resident aliens: the Tc1/mariner superfamily of transposable elements. Trends Genet 1999, 15(8):326-332.

29. Hey P, Robson G, Birch M, Bromley M: Characterisation of Aft1 a Fot1/ Pogo type transposon of Aspergillus fumigatus. Fungal Genet Biol 2008, 45(2):117-126

30. Nyyssonen E, Amutan M, Enfield L, Stubbs J, Dunn-Coleman NS: The transposable element Tan1 of Aspergillus niger var. awamori, a new member of the Fot1 family. Mol Gen Genet 1996, 253(1-2):50-56.

31. Dufresne M, Hua-Van A, El Wahab HA, Ben M'Barek S, Vasnier C, Teysset L, Kema GH, Daboussi MJ: Transposition of a fungal miniature invertedrepeat transposable element through the action of a Tc1-like transposase. Genetics 2007, 175(1):441-452.

32. Volff JN: Turning junk into gold: domestication of transposable elements and the creation of new genes in eukaryotes. Bioessays 2006 28(9):913-922.

33. Jurka J, Kapitonov W, Kohany O, Jurka MV: Repetitive sequences in complex genomes: structure and evolution. Annu Rev Genomics Hum Genet 2007, 8:241-259.

34. Biemont C, Vieira C: Genetics: junk DNA as an evolutionary force. Nature 2006, 443(7111):521-524

35. Siegmund T, Lehmann M: The Drosophila Pipsqueak protein defines a new family of helix-turn-helix DNA-binding proteins. Dev Genes Evol 2002, 212(3):152-157.

36. Wang $H$, Hartswood E, Finnegan DJ: Pogo transposase contains a putative helix-turn-helix DNA binding domain that recognises a $12 \mathrm{bp}$ sequence within the terminal inverted repeats. Nucleic Acids Res 1999, 27(2):455-461

37. Bigot Y, Brillet B, Auge-Gouillou C: Conservation of Palindromic and Mirror Motifs within Inverted Terminal Repeats of mariner-like Elements. J Mol Biol 2005, 351(1):108-116.

38. Krishna SS, Majumdar I, Grishin NV: Structural classification of zinc fingers: survey and summary. Nucleic Acids Res 2003, 31(2):532-550.

39. Ohta S, Tsuchida K, Choi S, Sekine Y, Shiga Y, Ohtsubo E: Presence of a characteristic D-D-E motif in IS1 transposase. J Bacteriol 2002, 184(22):6146-6154.

40. Feschotte C, Pritham EJ: DNA transposons and the evolution of eukaryotic genomes. Annu Rev Genet 2007, 41:331-368.

41. Molinari-Madlum EE, Felipe MS, Soares CM: Virulence of Paracoccidioides brasiliensis isolates can be correlated to groups defined by random amplified polymorphic DNA analysis. Med Mycol 1999, 37(4):269-276.

42. Miskey C, Izsvak Z, Kawakami K, Ivics Z: DNA transposons in vertebrate functional genomics. Cell Mol Life Sci 2005, 62(6):629-641.

43. Altschul SF, Gish W, Miller W, Myers EW, Lipman DJ: Basic local alignment search tool. J Mol Biol 1990, 215(3):403-410.

44. Rutherford K, Parkhill J, Crook J, Horsnell T, Rice P, Rajandream MA, Barrell B: Artemis: sequence visualization and annotation. Bioinformatics 2000 16(10):944-945. 
45. Ray DA, Feschotte C, Pagan HJ, Smith JD, Pritham EJ, Arensburger P, Atkinson PW, Craig NL: Multiple waves of recent DNA transposon activity in the bat, Myotis lucifugus. Genome Res 2008, 18(5):717-728.

46. Wang H, Liu JS: LTR retrotransposon landscape in Medicago truncatula: more rapid removal than in rice. BMC Genomics 2008, 9:382.

47. Kronmiller BA, Wise RP: TEnest: automated chronological annotation and visualization of nested plant transposable elements. Plant Physiol 2008, 146(1):45-59.

48. Thompson JD, Higgins DG, Gibson TJ: CLUSTAL W: improving the sensitivity of progressive multiple sequence alignment through sequence weighting, position-specific gap penalties and weight matrix choice. Nucleic Acids Res 1994, 22(22):4673-4680.

49. Galtier N, Gouy M, Gautier C: SEAVIEW and PHYLO_WIN: two graphic tools for sequence alignment and molecular phylogeny. Comput Appl Biosci 1996, 12(6):543-548.

50. Tamura K, Dudley J, Nei M, Kumar S: MEGA4: Molecular Evolutionary Genetics Analysis (MEGA) software version 4.0. Mol Biol Evol 2007, 24(8):1596-1599.

51. Girardin H, Latgé JP: DNA extraction and quantitation. Molecular Biology of Pathogenic fungi A Laboratory Manual New York: Telos PressMaresca B, Kobayashi GS 1994, 5-9.

52. Batista WL, Barros TF, Goldman GH, Morais FV, Puccia R: Identification of transcription elements in the $5^{\prime}$ intergenic region shared by LON and MDJ1 heat shock genes from the human pathogen Paracoccidioides brasiliensis. Evaluation of gene expression. Fungal Genet Biol 2007, 44(5):347-356.

doi:10.1186/1471-2164-11-130

Cite this article as: Marini et al:: Identification and characterization of TC1/mariner-like DNA transposons in genomes of the pathogenic fungi of the Paracoccidioides species complex. BMC Genomics 2010 11:130.

\section{Submit your next manuscript to BioMed Central and take full advantage of:}

- Convenient online submission

- Thorough peer review

- No space constraints or color figure charges

- Immediate publication on acceptance

- Inclusion in PubMed, CAS, Scopus and Google Scholar

- Research which is freely available for redistribution

Submit your manuscript at www.biomedcentral.com/submit
Biomed Central 